\title{
Quantization of projective homogeneous spaces and duality principle
}

\author{
Nicola Ciccoli, Rita Fioresi, and Fabio Gavarini*
}

\begin{abstract}
We introduce a general recipe to construct quantum projective homogeneous spaces, with a particular interest for the examples of the quantum Grassmannians and the quantum generalized flag varieties. Using this construction, we extend the quantum duality principle to quantum projective homogeneous spaces.
\end{abstract}

Mathematics Subject Classification (2000). Primary 20G42, 14M17; Secondary 17B37, 81R50. Keywords. Quantum homogeneous spaces.

\section{Contents}

1 Introduction . . . . . . . . . . . . . . . . . . . . . . . . . 449

2 The classical setting . . . . . . . . . . . . . . . . . . . 451

3 Quantum bundles and quantum homogeneous spaces . . . . . . . . . . . . . . . . 457

4 The quantum duality principle (QDP) . . . . . . . . . . . . . . . . . . . . 468

5 Examples: Quantum Grassmannians and quantum flag varieties . . . . . . . . . . 478

References . . . . . . . . . . . . . . . . . . . . . . . 494

\section{Introduction}

A projective variety can be described via its homogeneous graded coordinate ring. This ring is not an invariant associated to the variety but depends on a chosen embedding of the variety into some projective space. Different embeddings will, in general, produce non-isomorphic graded rings.

When a projective variety is homogeneous, i.e., endowed with a transitive action of an (affine) algebraic group on it, it can be realized as quotient of affine algebraic

\footnotetext{
${ }^{*} \mathrm{~N}$. Ciccoli was partially supported by TOK "Non commutative geometry and quantum groups", no. MKTD-CT-2004-509794, University of Warsaw; R. Fioresi and F. Gavarini were partially supported by the European RTN network "LIEGRITS - Flags, Quivers and Invariant Theory in Lie Representation Theory", no. MRTN-CT 2003-505078, and by the Italian research program PRIN 2005 "Moduli and Lie Theories".
} 
groups $G / H$. In this case a projective embedding can be obtained via sections of a line bundle on $G / H$, uniquely given once a character of $H$ is specified.

If one approaches a quantization of this picture in the context of quantum groups the problem immediately arising is that standard quantum groups have a very limited set of quantum subgroups. This explains why usually the preferred approach goes through representation theoretic techniques.

An explanation of the lack of quantum subgroups, together with a way to circumvent this problem, is suggested by considering the semiclassical picture, i.e., in the context of algebraic Poisson groups. In such setting algebraic Poisson subgroups are quite rare too; however there is no need of an algebraic Poisson subgroup to cook up a Poisson quotient. The existence of a surjective Poisson map $G \rightarrow G / H$ is guaranteed simply by requiring $H$ to be a coisotropic subgroup of $G$. This condition can be expressed by saying that the defining ideal of $H$, in the function algebra of $G$, is required to be a Poisson subalgebra rather than a Poisson ideal, as required for Poisson subgroups.

Let $\mathcal{O}_{q}(G)$ be a quantization of the affine algebraic Poisson groups $G$. At the quantum level, a quantization $\mathcal{O}_{q}(H)$ of its coisotropic subgroup $H$ can be defined through conditions on the projection $\pi: \mathcal{O}_{q}(G) \rightarrow \mathcal{O}_{q}(H)$. We will see this in full detail in the Sections 2, 3 .

Our first aim is to build a quantum deformation $\mathcal{O}_{q}(G / H)$ of the projective variety $G / H$, i.e., of its graded $\operatorname{ring} \mathcal{O}(G / H)$, subject to the following requirements:

(1) there exists a one-dimensional corepresentation of the quantum coisotropic subgroup $\mathcal{O}_{q}(H)$ which is a deformation of the corepresentation of $\mathcal{O}(H)$ corresponding to the character of $H$ which defines the line bundle giving the projective embedding of $G / H$;

(2) a quantum analogue $\mathcal{O}_{q}(G / H)$ to $\mathcal{O}(G / H)$ is defined as the subset - inside $\mathcal{O}_{q}(G)$ - of "semi-invariant functions" with respect to the given corepresentation of $\mathcal{O}_{q}(H)$

(3) the subset $\mathcal{O}_{q}(G / H)$ is a graded subalgebra of $\mathcal{O}_{q}(G)$;

(4) the graded subalgebra $\mathcal{O}_{q}(G / H)$ is a graded left coideal of $\mathcal{O}_{q}(G)$, so the coproduct in $\mathcal{O}_{q}(G)$ induces a (left) $\mathcal{O}_{q}(G)$-coaction on $\mathcal{O}_{q}(G / H)$, and the latter can be thought of as a quantum homogeneous space.

(5) the semiclassical limit of $\mathcal{O}_{q}(G / H)$ is $\mathcal{O}(G / H)$ - embedded into $\mathcal{O}(G)$ - as a graded subalgebra, left coideal and graded Poisson subalgebra.

In other words, a quantum deformation of a projective homogeneous space, embedded into some projective space, consists of the deformation of the graded algebra associated to the embedding in such a way that the action of the group on the homogeneous space is also naturally quantized.

We will work out the details of the construction for the case of the Grassmannian and its Plücker embedding, that is, when $G$ is the special linear group and $H=P$ 
is a maximal parabolic subgroup, and we will sketch it in the more general case of quantum flag varieties of simple Lie groups.

Our main motivation to develop this point of view is to adapt to projective homogeneous spaces the correspondence introduced by Ciccoli and Gavarini [5] for coinvariant subalgebras. This recipe allows to associate functorially to a quantum quasi-affine homogeneous space another quantum homogeneous space through a generalization of the quantum duality principle (QDP) defined by Drinfeld for quantum groups. A part of the arguments in [5] does not directly apply to projective homogeneous spaces, since it is based on the realization of the ring of the homogeneous space as the set of coinvariant functions inside the ring of the quantum group acting on it. But this is possible - as in the classical case - if and only if the homogeneous space is quasi-affine, which is not the case for projective varieties. The coordinate ring of the homogeneous space is replaced by a graded ring inside the quantum group ring consisting of semi-coinvariants with respect to a one-dimensional representation, which can be seen as a deformation of the line bundle that classically determines the projective embedding. The definitions introduced in Section 3 will allow us to define a quantum duality functor and to obtain the QDP construction in this more general setting. In the last chapter we will discuss applications to quantum flag manifolds.

\section{The classical setting}

In this section we recall some Poisson geometry (see [21] for details).

2.1. The affine case. Let $k$ be a fixed field of characteristic 0 . When doing algebraic geometry construction, we tacitly assume that $k$ be algebraically closed. However, this assumption is not needed for our quantum constructions.

Let $G$ be an affine algebraic group over k. We denote by $\mathcal{O}(G)$ the algebra of regular functions of $G$, in short its "function algebra", which is naturally a Hopf algebra (over k). We denote $g:=\operatorname{Lie}(G)$ the tangent Lie algebra of $G$, and similarly $\mathfrak{h}:=\operatorname{Lie}(H)$ for any closed algebraic subgroup $H$ of $G$.

Assume that $G$ is a Poisson group: this means that $\mathcal{O}(G)$ is a Poisson Hopf algebra, i.e., we have a Poisson bracket $\{\}:, \mathcal{O}(G) \otimes \mathcal{O}(G) \rightarrow \mathcal{O}(G)$ which is compatible with the Hopf algebra structure. Moreover, $g$ is a Lie bialgebra for some Lie cobracket $\delta: \mathfrak{g} \rightarrow \mathfrak{g} \otimes \mathfrak{g}$, and the same holds for its dual space $\mathfrak{g}^{*}$, these two Lie bialgebras structures being dual to each other. Indeed, the notion of Lie bialgebra is the infinitesimal counterpart of the notion of Poisson group. Since the dual $\mathrm{g}^{*}$ of $g$ is itself a Lie bialgebra, it follows that any connected algebraic group $G^{*}$ with $\mathrm{g}^{*}=\operatorname{Lie}\left(G^{*}\right)$ is a Poisson group on its own, called (Poisson) dual to $G$. We are going to see the example of the Poisson group $\mathrm{GL}_{n}$ treated in detail in Section 5.2. 
Definition 2.1. A (closed) subgroup $H$ of $G$ is called coisotropic if its defining ideal $I(H)$ is a Poisson subalgebra of $\mathcal{O}(G)$. Also, $H$ is called a Poisson subgroup if the embedding $H \hookrightarrow G$ is a Poisson map; this is equivalent to require $I(H)$ to be a Poisson ideal. Hence a Poisson subgroup is coisotropic.

The following equivalent conditions give an infinitesimal characterization for a connected subgroup $H$ to be coisotropic (see [24]):

Proposition 2.2. Let $G$ be an algebraic group and $H$ a (closed) subgroup of the Poisson group $G$. Then the following are equivalent:

(C-i) $H$ is a coisotropic subgroup of $G$;

(C-ii) $\delta(\mathfrak{h}) \subseteq \mathfrak{h} \wedge \mathfrak{g}$, that is, $\mathfrak{h}$ is (a Lie subalgebra and) a Lie coideal of $\mathfrak{g}$;

(C-iii) the orthogonal space $\mathfrak{h}^{\perp}$ is (a Lie coideal and) a Lie subalgebra of $\mathrm{g}^{*}$.

Remark 2.3. Note that, thanks to these characterizations, the infinitesimal counterpart of the notion of coisotropic subgroup is that of a Lie subalgebra Lie coideal. The latter notion is self-dual. In fact, let $G^{*}$ be any connected Poisson group dual to $G$. If $H$ is coisotropic in $G$, then any connected subgroup of $G^{*}$ with tangent Lie algebra $\mathfrak{h}^{\perp}$ is in turn a coisotropic subgroup of $G^{*}$, called "complementary dual" to $H$ and denoted by $H^{\perp}$.

We now want to describe the notion of Poisson quotient.

Definition 2.4. Let $M$ be a Poisson affine variety, i.e., an affine variety whose function algebra $\mathcal{O}(M)$ is a Poisson algebra. Then $M$ is a Poisson homogeneous $G$-space if there is a (regular) transitive action $\phi: G \times M \rightarrow M$ which is a Poisson map with respect to the product Poisson structure on $G \times M$. We say that a Poisson homogeneous $G$-space $M$ is a Poisson quotient if there is a coisotropic closed Lie subgroup $H_{M}$ of $G$ such that $G / H_{M} \simeq M$ and the projection $p_{M}: G \rightarrow G / H_{M} \simeq M$ is a Poisson map.

The following is a characterization of Poisson quotients (cf. [29]).

Proposition 2.5. Let $\phi: G \times M \rightarrow M$ be a homogeneous action of $G$ on $M$. Then the following are equivalent:

(PQ-i) there exists $\bar{m} \in M$ whose stabilizer $G_{\bar{m}}$ is a coisotropic subgroup of $G$;

(PQ-ii) there exists $\bar{m} \in M$ such that $\phi_{\bar{m}}: G \rightarrow M, g \mapsto \phi(g, \bar{m})$, is a Poisson map, that is to say, $M$ is a Poisson quotient;

(PQ-iii) there is $\bar{m} \in M$ such that $\{\bar{m}\}$ is a symplectic leaf of $M$. 
For any $\bar{m} \in M$ with stabilizer $G_{\bar{m}}$ one has $M \sim G / G_{\bar{m}}$ as affine $G$-varieties. As $M$ is affine, this is equivalent to $\mathcal{O}(M) \cong \mathcal{O}\left(G / G_{\bar{m}}\right)$. Finally, $\mathcal{O}\left(G / G_{\bar{m}}\right) \cong$ $\mathcal{O}(G)^{G_{\bar{m}}}$, the subalgebra of $G_{\bar{m}}$-invariants in $\mathcal{O}(G)$. The same holds with $G_{\bar{m}}$ replaced by any subgroup $H$ whose coset space $G / H$ is affine. We then have an additional characterization of (affine) Poisson quotients.

Proposition 2.6. If $M$ is as above, then $M$ is a Poisson quotient if and only if there exists $\bar{m} \in M$ such that $\mathcal{O}(G)^{G_{\bar{m}}}$ is a Poisson subalgebra of $\mathcal{O}(G)$.

In particular, if $H$ is a subgroup of $G$ and $G / H$ is affine, then the following are equivalent:

1. $H$ is coisotropic,

2. $G / H$ is a Poisson quotient,

3. $\mathcal{O}(G / H)=\mathcal{O}(G)^{H}$ is a Poisson subalgebra of $\mathcal{O}(G)$.

2.2. The projective case. We are now interested in the case when the homogeneous $G$-variety $G / H$ is projective, i.e., $H$ is parabolic. To describe this in algebraic terms (the setting we need for quantum deformations), we require a specific realization, namely an embedding into a projective space.

Given a representation $\rho$ of $H$ on some vector space $V$, we can construct a vector bundle associated to it, namely

$\mathcal{V}:=G \times_{H} V=G \times V / \simeq,(g h, v) \simeq\left(g, h^{-1} v\right), \quad$ for all $h \in H, g \in G, v \in V$.

The space of global sections of this bundle is identified with the induced module (see, e.g., [18] for more details)

$$
H^{0}(G / H, \mathcal{V})=\operatorname{Ind}_{H}^{G}(V)=\left\{f: G \rightarrow V \mid f \text { is regular, } f(g h)=h^{-1} \cdot f(g)\right\} .
$$

Definition 2.7. Let $\chi: H \rightarrow \mathbb{k}^{*}$ be a character of $H$, i.e., a one-dimensional representation of $H$ on $L \cong \mathbb{k}$. Then $L^{\otimes n}$ is again a one-dimensional representation of $H$ with character $\chi^{n}$. Let $\mathscr{L}^{n}:=G \otimes_{H} L^{\otimes n}$. Define

$$
\begin{aligned}
\mathcal{O}(G / H)_{n} & :=H^{0}\left(G / H, \mathscr{L}^{n}\right), \\
\mathcal{O}(G / H) & :=\bigoplus_{n \geq 0} \mathcal{O}(G / H)_{n} \subseteq \mathcal{O}(G) .
\end{aligned}
$$

Then $\mathcal{O}(G / H)$ is a subalgebra of $\mathcal{O}(G)$ whose elements are called semi-invariants. Note that now the notation $\mathcal{O}(G / H)$ has not the same meaning as when $G / H$ is affine.

Assume now the bundle $\mathscr{L}$ to be very ample. In the present context, this is the same (cf. [18], §II.7) as saying that $\mathscr{L}$ is generated by a set of global sections $f_{0}$, 
$f_{1}, \ldots, f_{N} \in \mathcal{O}(G / H)_{1}$; in particular, the algebra $\mathcal{O}(G / H)$ is graded, generated in degree 1 (by the $f_{i}$ 's). Then $\mathcal{O}(G / H)$ is the homogeneous coordinate ring of the projective variety $G / H$ with respect to the embedding given via the global sections of $\mathscr{L}$ (see [17], p. 176).

We want to reformulate this classical construction in purely Hopf algebraic terms, more suited to the quantum setting we shall presently deal with.

Remark 2.8. In algebraic terms, saying that $\chi: H \rightarrow \mathbb{k}^{*}$ is a character is the same as saying that it is a group-like element in the coalgebra $\mathcal{O}(H)$. The same holds for all powers $\chi^{n}(n \in \mathbb{N})$. In fact if $\chi$ is group-like, then the same is true for all its powers $\chi^{n}$ since $\mathcal{O}(H)$ is a Hopf algebra. As the $\chi^{n}$ 's are group-like, if they are pairwise different they also are linearly independent, which ensures that the sum $\sum_{n \in \mathbb{N}} \mathcal{O}(G / H)_{n}$ - inside $\mathcal{O}(G)$ - is a direct one. Moreover, once the embedding is given, each summand $\mathcal{O}(G / H)_{n}$ can be described in purely Hopf algebraic terms as

$$
\begin{aligned}
\mathcal{O}(G / H)_{n} & :=\left\{f \in \mathcal{O}(G) \mid f(g h)=\chi^{n}\left(h^{-1}\right) f(g)\right\} \\
& =\left\{f \in \mathcal{O}(G) \mid((\mathrm{id} \otimes \pi) \circ \Delta)(f)=f \otimes S\left(\chi^{n}\right)\right\},
\end{aligned}
$$

with $\pi: \mathcal{O}(G) \rightarrow \mathcal{O}(H)$ the standard projection and $S$ the antipode of $\mathcal{O}(H)$.

To simplify notation, we set $\lambda:=S(\chi)$, the character of $H$ which maps $h \in H$ to $\lambda(h)=\chi\left(h^{-1}\right)$, and we set $\Delta_{\pi}:=($ id $\otimes \pi) \circ \Delta$, so that

$$
\mathcal{O}(G / H)_{n}=\left\{f \in \mathcal{O}(G) \mid \Delta_{\pi}(f)=f \otimes \lambda^{n}\right\} .
$$

Proposition 2.9. Let $G / H$ be embedded into some projective space via some very ample line bundle. Then there exists a $t \in \mathcal{O}(G)$ such that

$$
\begin{aligned}
\Delta_{\pi}(t) & :=((\mathrm{id} \otimes \pi) \circ \Delta)(t)=t \otimes \pi(t), \\
\pi\left(t^{m}\right) & \neq \pi\left(t^{n}\right) \text { for all } m \neq n \in \mathbb{N}, \\
\mathcal{O}(G / H)_{n} & =\left\{f \in \mathcal{O}(G) \mid \Delta_{\pi}(f)=f \otimes \pi\left(t^{n}\right)\right\}, \\
\mathcal{O}(G / H) & =\bigoplus_{n \in \mathbb{N}} \mathcal{O}(G / H)_{n},
\end{aligned}
$$

and $\mathcal{O}(G / H)$ is generated in degree 1 , namely by $\mathcal{O}(G / H)_{1}$.

Proof. If $f \in \mathcal{O}(G / H)_{n}$, then

$$
\pi(f)=\pi\left(\sum_{(f)} \epsilon\left(f_{(1)}\right) f_{(2)}\right)=(\epsilon \otimes \pi)(\Delta(f))=(\epsilon \otimes \mathrm{id})\left(\Delta_{\pi}(f)\right)=\epsilon(f) \lambda^{n} .
$$

Now by assumption there exists a non-zero global section of the line bundle on $G / H$, i.e., a regular function $t \in \mathcal{O}(G / H)_{1} \backslash\{0\}$ on $G$ and $\epsilon(t) \neq 0$. By the above (for $n=1$ ), up to dividing out by $\epsilon(t)$, we can assume that $\pi(t)=\lambda$. The result follows immediately. 
Notice that while $\lambda=\pi(t)$ is group-like, $t$ instead is something less, yet still has an "almost group-like property" given by (2.2). This element $t$ and its quantization will turn out to be crucial for the quantum setting.

Remark 2.10. We point out that $\mathcal{O}(G / H)$ is a unital subalgebra and also a (left) coideal of $\mathcal{O}(G)$; the latter reflects the fact that $G / H$ is a (left) $G$-space. Thus, the restriction of the comultiplication of $\mathcal{O}(G)$, namely

$$
\left.\Delta\right|_{\mathcal{O}(G / H)}: \mathcal{O}(G / H) \rightarrow \mathcal{O}(G) \otimes \mathcal{O}(G / H),
$$

is a coaction of $\mathcal{O}(G)$ on $\mathcal{O}(G / H)$, which makes $\mathcal{O}(G / H)$ into an $\mathcal{O}(G)$-comodule algebra in the sense of [26], §4.1. Moreover $\mathcal{O}(G / H)$ is graded and the coaction $\left.\Delta\right|_{\mathcal{O}(G / H)}$ is also graded with respect to the trivial grading on $\mathcal{O}(G)$, so that each $\mathcal{O}(G / H)_{n}$ is indeed a coideal of $\mathcal{O}(G)$ as well.

As to Poisson geometrical properties in this projective setup, the following characterization, which might be used to define the notion of Poisson quotient structure for the projective $G$-space $G / H$, holds:

Proposition 2.11. Let $G$ be a Poisson algebraic group, $H$ a closed parabolic subgroup, and $t \in \mathcal{O}(G)$ as in Remark 2.8. The following are equivalent:

(a) $\{I(H), I(H)\} \subseteq I(H)$, that is, $H$ is coisotropic, and in addition $\{t, \mathcal{O}(G / H)\} \subseteq$ $I(H)$;

(b) $\left\{\mathcal{O}(G / H)_{r}, \mathcal{O}(G / H)_{s}\right\} \subseteq \mathcal{O}(G / H)_{r+s}$ for all $r, s \in \mathbb{N}$, that is, $\mathcal{O}(G / H)$ is a graded Poisson subalgebra of $\mathcal{O}(G)$.

Proof. To simplify notation, we set $I_{H, n}:=I(H) \cap \mathcal{O}(G / H)_{n}$ for $n \in \mathbb{N}$.

(a) $\Longrightarrow$ (b): First of all, note that (2.1) can be reformulated as

$$
\mathcal{O}(G / H)_{n}=\left\{f \in \mathcal{O}(G) \mid \Delta(f) \in f \otimes t^{n}+\mathcal{O}(G) \otimes I(H)\right\} .
$$

Second, by Remark $2.10(\mathrm{a})$, each $\mathcal{O}(G / H)_{n}$ is a coideal of $\mathcal{O}(G)$, that is, $\Delta\left(\mathcal{O}(G / H)_{n}\right) \subseteq \mathcal{O}(G) \otimes \mathcal{O}(G / H)_{n}$. This along with (2.6) gives

$$
\Delta(f) \in f \otimes t^{n}+\mathcal{O}(G) \otimes I_{H, n} \quad \text { for all } f \in \mathcal{O}(G / H)_{n} .
$$

Then, for any $f \in \mathcal{O}(G / H)_{r}$ and $\ell \in \mathcal{O}(G / H)_{s}$, we have

$$
\begin{aligned}
\Delta(\{f, \ell\})= & \Delta(f), \Delta(\ell)\} \in\left\{f \otimes t^{r}+\mathcal{O}(G) \otimes I_{H, r}, \ell \otimes t^{s}+\mathcal{O}(G) \otimes I_{H, s}\right\} \\
= & \left\{f \otimes t^{r}, \ell \otimes t^{s}\right\}+\left\{f \otimes t^{r}, \mathcal{O}(G) \otimes I_{H, s}\right\} \\
& +\left\{\mathcal{O}(G) \otimes I_{H, r}, \ell \otimes t^{s}\right\}+\left\{\mathcal{O}(G) \otimes I_{H, r}, \mathcal{O}(G) \otimes I_{H, s}\right\} \\
\subseteq & f f,\} \otimes t^{r+s}+f \ell \otimes\left\{t^{r}, t^{s}\right\}+\{f, \mathcal{O}(G)\} \otimes t^{r} I_{H, s} \\
& +f \mathcal{O}(G) \otimes\left\{t^{r}, I_{H, s}\right\}+\{\mathcal{O}(G), \ell\} \otimes I_{H, r} t^{s}+\mathcal{O}(G) \ell \otimes\left\{I_{H, r}, t^{s}\right\} \\
& +\{\mathcal{O}(G), \mathcal{O}(G)\} \otimes I_{H, r} I_{H, s}+\mathcal{O}(G) \mathcal{O}(G) \otimes\left\{I_{H, r}, I_{H, s}\right\} \\
\subseteq & \{f, \ell\} \otimes t^{r+s}+\mathcal{O}(G) \otimes I_{H, r+s}
\end{aligned}
$$


due to (2.7) and to (a). Thus $\{f, \ell\} \in \mathcal{O}(G / H)_{r+s}$ by (2.6) again.

(b) $\Longrightarrow$ (a): By assumption we have $t \in \mathcal{O}(G / H)_{1}$, hence $\{t, f\} \in \mathcal{O}(G / H)_{1+n}$ for all $f \in \mathcal{O}(G / H)_{n}$ by (b). In particular, this gives

$$
\{t, f\}(h)=t^{1+n}(h)\{t, f\}\left(1_{G}\right)=0 \quad \text { for all } h \in H
$$

because any Poisson group structure is zero at the identity. Eventually, this yields that $\{t, \mathcal{O}(G / H)\} \subseteq I(H)$.

To prove that $\{I(H), I(H)\} \subseteq I(H)$, we need some additional tools.

First, let $\mathcal{O}(G)_{1_{G}}$ be the localization of $\mathcal{O}(G)$ at $J:=\operatorname{Ker}\left(\epsilon_{\mathcal{O}(G)}\right)$, a maximal ideal in $\mathcal{O}(G)$. This is the stalk at the point $1_{G}$ of the structure sheaf of $G$, and the Poisson bracket of $\mathcal{O}(G)$ canonically (and uniquely) extends, for $f_{1}, f_{2} \in \mathcal{O}(G)$, $y_{1}, y_{2} \in J, n_{1}, n_{2} \in \mathbb{N}$, via the identity

$$
\begin{aligned}
\left\{f_{1} y_{1}^{-n_{1}}, f_{2} y_{2}^{-n_{2}}\right\}= & \left\{f_{1}, f_{2}\right\} y_{1}^{-n_{1}} y_{2}^{-n_{2}}-n_{1} f_{1} y_{1}^{-n_{1}-1}\left\{y_{1}, f_{2}\right\} y_{2}^{-n_{2}} \\
& -n_{2} f_{2}\left\{f_{1}, f_{2}\right\} y_{1}^{-n_{1}} y_{2}^{-n_{2}-1}+n_{1} n_{2} f_{1} f_{2}\left\{y_{1}, y_{2}\right\} y_{1}^{n_{1}-1} y_{2}^{n_{2}-1}
\end{aligned}
$$

to the local algebra $\mathcal{O}(G)_{1_{G}}$. The counit $\epsilon$ of $\mathcal{O}(G)$ uniquely extends to an algebra morphism from $\mathcal{O}(G)_{1_{G}}$ to $\mathbb{k}$, again denoted by $\epsilon$, whose kernel is $J_{1_{G}}$, the localization of $J$ inside $\mathcal{O}(G)_{1_{G}}$. Finally, we denote by $I(H)_{1_{G}}$ the localization, inside $\mathcal{O}(G)_{1_{G}}$, of the ideal $I(H)$ of $\mathcal{O}(G)$.

Second, let $X_{t}:=\{t=0\}$ be the zero locus in $G / H$ defined by the vanishing of the divisor $t$, and let $\Gamma:=(G / H) \backslash X_{t}$. This is an affine open dense subset of $G / H$ whose algebra of regular functions is the graded localization of $\mathcal{O}(G / H)$ by the multiplicative subset $\left\{t^{n}\right\}_{n \in \mathbb{N}}$, that is, $\mathcal{O}(G / H)_{[t]}:=\bigoplus_{n \in \mathbb{N}} t^{-n} \mathcal{O}(G / H)_{n}$. Note that $\mathcal{O}(G / H)_{[t]}$ naturally embeds into $\mathcal{O}(G)_{1_{G}}$ because $t \in J^{G / H}:=\mathcal{O}(G / H) \backslash J$. Again, the Poisson bracket of $\mathcal{O}(G / H)$, induced by that of $\mathcal{O}(G)$, uniquely extends to $\mathcal{O}(G / H)_{[t]}$, and so the latter is a graded Poisson subalgebra of $\mathcal{O}(G)_{1_{G}}$; thus $X_{t}$ is an affine Poisson variety. Also, $\epsilon$ induces an algebra morphism from $\mathcal{O}(G / H)_{[t]}$ to $\mathbb{k}$, whose kernel we denote by $J_{[t]}^{G / H}$.

Third, let $\mathcal{O}(G / H)_{\overline{1_{G}}}$ be the localization of $\mathcal{O}(G / H)_{[t]}$ at $J_{[t]}^{G / H}$ : by construction, this is the stalk at $\overline{1_{G}}=1_{G} H$ of the structure sheaf of $G / H$, and the Poisson bracket of $\mathcal{O}(G / H)_{[t]}$ uniquely extends to $\mathcal{O}(G / H)_{\overline{1_{G}}}$.

Now the maximal ideal $J_{1_{G}}$ in the local algebra $\mathcal{O}(G)_{1_{G}}$ can be generated by a local system of parameters on $G$ at the point $1_{G}$, say $\left\{y_{1}, \ldots, y_{n}\right\}$, with $n:=\operatorname{dim}(G)$. As $H$ is a closed subgroup of $G$, we can choose this system of parameters in such a way that:

(1) if $h:=\operatorname{dim}(H)$, the image inside $\mathcal{O}(H)_{1_{H}} \cong \mathcal{O}(G)_{1_{G}} / I(H)_{1_{G}}$ of $\left\{y_{1}, \ldots, y_{h}\right\}$ is a local system of parameters on $H$ at the point $1_{H}=1_{G}$;

(2) $y_{h}=t-1$; 
(3) $\left\{y_{h+1}, \ldots, y_{n}\right\}$ is a local system of parameters on $X_{t}$ at $1_{G}$; in particular, it generates in $\mathcal{O}(G / H)_{\overline{1}}$ the ideal $J_{\frac{G / H}{1_{G}}}:=\operatorname{Ker}(\epsilon) \cap \mathcal{O}(G / H)_{\overline{1_{G}}}$.

As a direct consequence of the above assumptions, the elements $y_{h+1}, \ldots, y_{n}$ generate the ideal $I(H)_{1_{G}}$ inside $\mathcal{O}(G)_{1_{G}}$. Moreover, we have $y_{i}=f_{i} \ell_{i}^{-1}$ for some $f_{i} \in \mathcal{O}(G / H), \ell_{i} \in \mathcal{O}(G / H) \backslash J^{G / H}$ (for $\left.i=h+1, \ldots, n\right)$. Then

$$
\begin{aligned}
\left\{y_{i}, y_{j}\right\}=\left\{f_{i} \ell_{i}^{-1}, f_{j} \ell_{j}^{-1}\right\}=\{ & \left.f_{i}, f_{j}\right\} \ell_{i}^{-1} \ell_{j}^{-1}-\left\{f_{i}, \ell_{j}\right\} \ell_{i}^{-1} f_{j} \ell_{j}^{-2} \\
& -f_{i}\left\{\ell_{i}, f_{j}\right\} \ell_{i}^{-2} \ell_{j}^{-1}+f_{i} f_{j}\left\{\ell_{i}^{-1}, \ell_{j}^{-1}\right\} \ell_{i}^{-2} \ell_{j}^{-2}
\end{aligned}
$$

which - by assumption (b) yielding $\{\mathcal{O}(G / H), \mathcal{O}(G / H)\} \subseteq \mathcal{O}(G / H)$ - shows that $\left\{y_{i}, y_{j}\right\} \in\left(y_{h+1}, \ldots, y_{n}\right)=I(H)_{1_{G}}$ for all $i, j$. This together with Leibniz's rule implies that $\left\{k_{i} y_{i}, k_{j} y_{j}\right\} \in\left(y_{h+1}, \ldots, y_{n}\right)=I(H)_{1_{G}}$ for any $k_{i}, k_{j} \in$ $\mathcal{O}(G)_{1_{G}}$ (with $\left.i, j=h+1, \ldots, n\right)$; in turn, $I(H)_{1_{G}}=\left(y_{h+1}, \ldots, y_{n}\right)$ satisfies $\left\{I(H)_{1_{G}}, I(H)_{1_{G}}\right\} \subseteq I(H)_{1_{G}}$.

Eventually, since $I(H)=\mathcal{O}(G) \cap I(H)_{1_{G}}$, the above results also give

$$
\{I(H), I(H)\} \subseteq \mathcal{O}(G) \cap\left\{I(H)_{1_{G}}, I(H)_{1_{G}}\right\} \subseteq \mathcal{O}(G) \cap I(H)_{1_{G}}=I(H) .
$$

\section{Quantum bundles and quantum homogeneous spaces}

3.1. Quantum groups. We want to translate all the framework of Section 2 into the quantum setup. The first step is to introduce quantum groups, in the form of quantum (or "quantized") function algebras, as follows.

Let $G$ be an algebraic Poisson group and $\mathcal{O}(G)$ its function algebra.

Definition 3.1. By quantization of $\mathcal{O}(G)$ we mean a Hopf algebra $\mathcal{O}_{q}(G)$ over the ground ring $\mathbb{k}_{q}:=\mathbb{k}\left[q, q^{-1}\right]$, where $q$ is an indeterminate such that:

(a) the specialization of $\mathcal{O}_{q}(G)$ at $q=1$, that is, $\mathcal{O}_{q}(G) /(q-1) \mathcal{O}_{q}(G)$, is isomorphic to $\mathcal{O}(G)$ as a Poisson Hopf algebra;

(b) $\mathcal{O}_{q}(G)$ is torsion-free as a $\mathbb{k}_{q}$-module;

(c) if $I_{G}:=(q-1) \mathcal{O}_{q}(G)+\operatorname{Ker}\left(\epsilon_{\mathcal{O}_{q}(G)}\right)$, then $\bigcap_{n \geq 0} I_{G}^{n}=\bigcap_{n \geq 0}(q-1)^{n} \mathcal{O}_{q}(G)$.

We call $\mathcal{O}_{q}(G)$ quantum (or quantized) function algebra over $G$, or quantum deformation of $G$, or even simply quantum group. It is standard terminology to say that the Poisson Hopf algebra $\mathcal{O}(G)$ is the semiclassical limit of $\mathcal{O}_{q}(G)$.

Similarly, we say that a $\mathrm{k}_{q}$-algebra $\mathcal{O}_{q}(X)$ is a quantization of the commutative k-algebra $\mathcal{O}(X)$ if it is torsion-free and $\mathcal{O}_{q}(X) /(q-1) \mathcal{O}_{q}(X) \cong \mathcal{O}(X)$. Then $\mathcal{O}(X)$ is also a Poisson algebra, called semiclassical limit of $\mathcal{O}_{q}(X)$. 
Remark 3.2. (1) The technical requirement in (c) corresponds, in the context of formal deformations, to require that the algebra is separated; we also point out that it is satisfied by all quantum function algebras usually considered in literature. In any case, it will not be necessary until Section 5. Moreover, both (b) and (c) above are automatically satisfied when $\mathcal{O}_{q}(G)$ is free as a $\mathbb{k}_{q}$-module.

(2) The classical algebra $\mathcal{O}(G)$ inherits from $\mathcal{O}_{q}(G)$ a Poisson bracket given as follows: if $x, y \in \mathcal{O}_{q}(G) /(q-1) \mathcal{O}_{q}(G) \cong \mathcal{O}(G)$, then

$$
\{x, y\}:=\frac{x^{\prime} y^{\prime}-y^{\prime} x^{\prime}}{q-1} \bmod (q-1) \mathcal{O}_{q}(G)
$$

for any lifts $x^{\prime}, y^{\prime} \in \mathcal{O}_{q}(G)$ of $x$ and $y$, respectively. One checks that this bracket is well defined and makes $\mathcal{O}(G)$ into a Poisson Hopf algebra, so that $G$ is a Poisson group. But $G$ already had, by assumption, a Poisson group structure; then the requirement in (a) above that $\mathcal{O}_{q}(G) /(q-1) \mathcal{O}_{q}(G) \cong \mathcal{O}(G)$ as Poisson Hopf algebra amounts to say that, in particular, the two Poisson group structures of $G$ are isomorphic.

On the other hand, if we start without asking $G$ to have a Poisson group structure, then the previous analysis tells that if a quantization $\mathcal{O}_{q}(G)$ exists, then it automatically endows $\mathcal{O}(G)$ with a Poisson algebra structure. And similarly for a quantization $\mathcal{O}_{q}(X)$ of a commutative algebra $\mathcal{O}(X)$.

3.2. Quantum subgroups and quantum coisotropic subgroups. Our second step is to introduce the notions of quantum coisotropic subgroup and of quantum subgroup, the former being weaker than the latter.

Definition 3.3. By quantum coisotropic subgroup of $\mathcal{O}_{q}(G)$ we mean a $\mathbb{k}_{q}$-coalgebra $\mathcal{O}_{q}(H)$, along with a projection $\pi: \mathcal{O}_{q}(G) \rightarrow \mathcal{O}_{q}(H)$ such that

(a) $\mathcal{O}_{q}(H)$ is torsion-free, as a $\mathbb{k}_{q}$-module;

(b) $\pi$ is a $\mathbb{k}_{q}$-coalgebra (epi)morphism;

(c) $\pi$ is an $\mathcal{O}_{q}(G)$-module (epi)morphism, where $\mathcal{O}_{q}(H)$ has the $\mathcal{O}_{q}(G)$-module structure induced by $\pi$, that is, $f \cdot \pi(g)=\pi(f g)$.

If, in addition, $\mathcal{O}_{q}(H)$ is a Hopf algebra and $\pi$ is a Hopf algebra morphism, and for $I_{H}:=(q-1) \mathcal{O}_{q}(H)+\operatorname{Ker}\left(\epsilon_{\mathcal{O}_{q}(H)}\right)$ we have

$$
\bigcap_{n \geq 0} I_{H}^{n}=\bigcap_{n \geq 0}(q-1)^{n} \mathcal{O}_{q}(H)
$$

then we say that $\mathcal{O}_{q}(H)$ is a quantum subgroup of $G$.

For later use, we introduce also the notation $I_{q}(H):=\operatorname{Ker}(\pi)$.

Remark 3.4. (1) $I_{q}(H):=\operatorname{Ker}(\pi)$ satisfies $I_{q}(H) \cap(q-1) \mathcal{O}_{q}(G)=(q-1) I_{q}(H)$. So the specialization of $I_{q}(H)$ at $q=1$, i.e., $I_{1}(H):=I_{q}(H) /(q-1) I_{q}(H)$, 
coincides with the image of $I_{q}(H)$ under the specialization $\mathcal{O}_{q}(G) /(q-1) \mathcal{O}_{q}(G) \cong$ $\mathcal{O}(G)$ of $\mathcal{O}_{q}(G)$, which is $I_{q}(H) /\left(I_{q}(H) \cap(q-1) \mathcal{O}_{q}(G)\right)$.

(2) Conditions (b) and (c) imply that $I_{q}(H)$ is a two-sided coideal and a left ideal of $\mathcal{O}_{q}(G)$. Then, by (1), the specialization $I_{1}(H)$ is a coideal and a (two-sided) ideal in the commutative ring $\mathcal{O}(G)$. Moreover, $I_{1}(H)$ equals the kernel of $\pi_{1}: \mathcal{O}(G)=$ $\mathcal{O}_{1}(G) \rightarrow \mathcal{O}_{1}(H)$, the specialization of $\pi$ at $q=1$, where $\mathcal{O}_{1}(H):=\mathcal{O}_{q}(H) /(q-$ 1) $\mathcal{O}_{q}(H)$ is the specialization of $\mathcal{O}_{q}(H)$. So $\mathcal{O}_{1}(H)$ admits the unique quotient Hopf algebra structure such that $\pi_{1}$ is the canonical Hopf algebra epimorphism. In particular, $\mathcal{O}_{1}(H)$ is the function algebra $\mathcal{O}(H)$ of some closed algebraic subgroup $H$ of $G$, and $I_{1}(H)=\operatorname{Ker}\left(\pi_{1}: \mathcal{O}(G) \rightarrow \mathcal{O}(H)\right)=I(H)$, whence the terminology and notation.

In the Hopf algebra language, conditions (b) and (c) are expressed by saying that $\mathcal{O}_{q}(H)$ is an $\mathcal{O}_{q}(G)$-module coalgebra, that is, a coalgebra and $\mathcal{O}_{q}(G)$-module such that $\Delta_{\mathcal{O}_{q}(H)}$ and $\epsilon_{\mathcal{O}_{q}(H)}$ are $\mathcal{O}_{q}(G)$-module morphisms.

(3) Assumptions at the quantum level imply properties for specializations. So the semiclassical specialization of a quantum coisotropic subgroup is (the function algebra of) a coisotropic subgroup since $I_{1}(H)=\operatorname{Ker}\left(\pi_{1}\right)$ is a Poisson subalgebra of $\mathcal{O}(G)$. On the other hand, the specialization of a quantum subgroup instead is (the function algebra of) a Poisson subgroup.

At the semiclassical level there are many examples of coisotropic subgroups, among which only a few are Poisson subgroups. This is a key motivation to focus on the more general setting of quantum coisotropic subgroups.

(4) A quantum coisotropic subgroup $\mathcal{O}_{q}(H)$ is by no means a "quantum group" in the sense of Definition 3.1, unless it is a quantum subgroup.

3.3. Quantum line bundles. We now want to carry over to the quantum setting the notion of embedding $G / H \hookrightarrow \mathbb{P}^{N}$ associated to a line bundle $\mathscr{L}$ that we assume to be very ample. The idea is to transfer to this framework the description (2.4) of $\mathcal{O}(G / H)_{n}$ given in terms of an element $t \in \mathcal{O}(G)$, as in Remark 2.8 and Proposition 2.9. Thus, the starting point will be a quantization of such an element $t$ that we will call a pre-quantum section.

Given $G$ and $H$ as in Section 2.2, we assume that quantizations of them be given, i.e., we are given $\mathcal{O}_{q}(G), \mathcal{O}_{q}(H)$ and $\pi: \mathcal{O}_{q}(G) \rightarrow \mathcal{O}_{q}(H)$ as in Definitions 3.1, 3.3. To simplify notation, hereafter we shall also write $\bar{\ell}:=\pi(\ell) \in \mathcal{O}_{q}(H)$ for every $\ell \in \mathcal{O}_{q}(G)$.

Moreover, we assume that an element $t \in \mathcal{O}(G)$ as in Remark 2.8, and the corresponding closed embedding $G / H \hookrightarrow \mathbb{P}^{N}$ be given as in Proposition 2.9 (so, in particular, $t$ is a section of the line bundle $\mathscr{L}$ on $G / H$ ).

We define a quantization of the latter setup as follows.

Definition 3.5. We define pre-quantization of $t$ or pre-quantum section of the line bundle $\mathscr{L}$ on $G / H$ (given by $t$ ) any $d \in \mathcal{O}_{q}(G)$ such that 
(a) $\Delta_{\pi}(d)=d \otimes \pi(d)$, i.e., $\Delta(d) \in\left(d \otimes d+\mathcal{O}_{q}(G) \otimes I_{q}(H)\right)$,

(b) $d \bmod (q-1) \mathcal{O}_{q}(G)=t(\in \mathcal{O}(G))$

with respect to the identification $\mathcal{O}_{q}(G) /(q-1) \mathcal{O}_{q}(G) \cong \mathcal{O}(G)$.

Remark 3.6. (a) Given a pre-quantum section $d$, property (a) in Definition 3.5 implies that $\pi(d)=\bar{d}$ is a group-like element in $\mathcal{O}_{q}(H)$. Therefore, it defines a onedimensional corepresentation of $\mathcal{O}_{q}(H)$, namely

$$
\rho_{d}: \mathbb{k}_{q} \rightarrow \mathbb{k}_{q} \otimes_{\mathbb{k}_{q}} \mathcal{O}_{q}(H), \quad 1 \mapsto 1 \otimes \bar{d},
$$

which gives back, modulo $(q-1)$, the one-dimensional representation of $\mathcal{O}(H)$,

$$
\rho_{\lambda}: \mathbb{k} \rightarrow \mathbb{k} \otimes_{\mathbb{k}} \mathcal{O}(H), \quad 1 \mapsto 1 \otimes \lambda,
$$

corresponding to the character $\lambda=\pi(t)$ of the group $H$ we started from.

(b) In the classical setup, having the character $\lambda$ is equivalent to having a Hopf algebra morphism $\mathbb{k}\left[x, x^{-1}\right] \rightarrow \mathcal{O}(H)$ given by $x^{z} \mapsto \lambda^{z}(z \in \mathbb{Z})$. Indeed, this occurs because the powers $\lambda^{z}$ do exist in $\mathcal{O}(H)$ and are group-like because $\lambda$ is. In fact, each one of them corresponds to a one-dimensional corepresentation, namely the $z$-th tensor power of $\rho_{\lambda}$,

$$
\rho_{\lambda}^{\otimes z}=\rho_{\lambda^{z}}: \mathbb{k} \rightarrow \mathbb{k} \otimes_{\mathbb{k}} \mathcal{O}(H), \quad 1 \mapsto 1 \otimes \lambda^{z} .
$$

On the other hand, in the quantum setup there is no natural analogue, since the powers $\bar{d}^{z}$ are not even defined in $\mathcal{O}_{q}(H)$ - which is not an algebra! - nor can we assume (in case we define them in some way) that they are group-like. This means that we miss somehow the "tensor powers" of $\rho_{d}$. In [1] one can find an example of a countable family of group-like elements in a quantum coisotropic subgroup, which are not obtained by projecting powers of the same element, but quantize a classical character.

However, when $\mathcal{O}_{q}(H)$ is a quantum subgroup instead, it is a Hopf algebra; hence the group-like $\bar{d}$ is invertible, and all powers $\bar{d}^{z}$ exist and are group-like in $\mathcal{O}_{q}(H)$. So we do have all "tensor power corepresentations"

$$
\rho_{d}^{\otimes z}: \mathbb{k}_{q} \rightarrow \mathbb{k}_{q} \otimes_{\mathbb{k}_{q}} \mathcal{O}_{q}(H), \quad 1 \mapsto 1 \otimes \bar{d}^{z},
$$

which in turn means that having $\bar{d}$ is equivalent to having a Hopf $\mathbb{k}_{q}$-algebra morphism $\mathbb{k}_{q}\left[x, x^{-1}\right] \rightarrow \mathcal{O}_{q}(H)$ given by $x^{z} \mapsto \bar{d}^{z}(z \in \mathbb{Z})$.

Moreover, notice also that $\bar{d}^{z}=\bar{d}^{z}$ for all $z \geq 0$, so that $\rho_{d}^{\otimes z}$ for $z \geq 0$ can be directly recovered from the element $d^{z}$ in $\mathcal{O}_{q}(G)$; thus in the end we can handle everything working with the elements $d^{n} \in \mathcal{O}_{q}(G), n \in \mathbb{N}$. 
Definition 3.7. Let $d \in \mathcal{O}_{q}(G)$ be a pre-quantum section on $G / H$.

(a) We call $d$-semi-invariants of degree $n$ the elements of the set

$$
\begin{aligned}
\mathcal{O}_{q}(G / H)_{n} & :=\left\{\ell \in \mathcal{O}_{q}(G) \mid \Delta_{\pi}(\ell)=\ell \otimes \pi\left(d^{n}\right)\right\} \\
& =\left\{\ell \in \mathcal{O}_{q}(G) \mid \Delta(\ell) \in\left(\ell \otimes d^{n}+\mathcal{O}_{q}(G) \otimes I_{q}(H)\right)\right\}
\end{aligned}
$$

(b) We call $d$-semi-invariants the elements of the set

$$
\mathcal{O}_{q}(G / H):=\sum_{n \in \mathbb{N}} \mathcal{O}_{q}(G / H)_{n}
$$

It is clear that each $\mathcal{O}_{q}(G / H)_{n}$ is a $\mathbb{k}_{q}$-submodule of $\mathcal{O}_{q}(G)$, hence the same holds for $\mathcal{O}_{q}(G / H)$. We shall now see some further properties of these modules, which eventually will tell us that - under suitable, additional assumptions - we can take $\mathcal{O}_{q}(G / H)$ as a quantization of $\mathcal{O}(G / H)$.

Lemma 3.8. Let $d \in \mathcal{O}_{q}(G)$ be a pre-quantum section on $G / H$. Then:

(a) $d \in \mathcal{O}_{q}(G / H)_{1}$, i.e., $d$ is semi-invariant of degree 1 .

(b) for any $n \in \mathbb{N}$ and any $\ell \in \mathcal{O}_{q}(G / H)_{n}$, we have $\bar{\ell}=\epsilon(\ell) \overline{d^{n}}$.

(c) the map $\pi: \mathcal{O}_{q}(G) \rightarrow \mathcal{O}_{q}(H)$ restricts to a $\mathbb{k}_{q}$-module epimorphism

$$
\pi^{\prime}: \mathcal{O}_{q}(G / H) \rightarrow \operatorname{span}_{\mathbb{k}_{q}}\left(\left\{\overline{d^{n}}\right\}_{n \in \mathbb{N}}\right) .
$$

Proof. The only statement which needs a proof here is (b), which quickly follows applying $(\epsilon \otimes \mathrm{id})$ to both sides of $\Delta_{\pi}(\ell)=\ell \otimes \overline{d^{n}}$.

Remark 3.9. The semi-invariants have a good arithmetic property, which ensures that the specialization of $\mathcal{O}_{q}(G / H)_{n} \subset \mathcal{O}_{q}(G)$ (and of $\mathcal{O}_{q}(G / H)$ ) at $q=1$ will be consistent with that of $\mathcal{O}_{q}(G)$ itself. Namely, given $n \in \mathbb{N}$, since $\mathcal{O}_{q}(G / H)_{n}$ is defined by $\mathbb{k}_{q}$-linear conditions we find at once that

$$
\mathcal{O}_{q}(G / H)_{n} \cap c \mathcal{O}_{q}(G)=c \mathcal{O}_{q}(G / H)_{n} \quad \text { for all } c \in \mathbb{k}_{q},
$$

and, in particular, $\mathcal{O}_{q}(G / H)_{n} \cap(q-1) \mathcal{O}_{q}(G)=(q-1) \mathcal{O}_{q}(G / H)_{n}$.

The next result shows that each $\mathcal{O}_{q}(G / H)_{n}$ is a left coideal of $\mathcal{O}_{q}(G)$, hence it bears a structure of left $\mathcal{O}_{q}(G)$-comodule.

Proposition 3.10. Every $\mathcal{O}_{q}(G / H)_{n}$ is a left coideal of $\mathcal{O}_{q}(G)$, that is,

$$
\Delta\left(\mathcal{O}_{q}(G / H)_{n}\right) \subseteq \mathcal{O}_{q}(G) \otimes \mathcal{O}_{q}(G / H)_{n} \quad \text { for all } n \in \mathbb{N}
$$

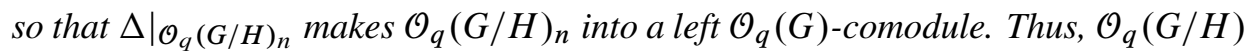
is a left coideal of $\mathcal{O}_{q}(G)$, hence a left $\mathcal{O}_{q}(G)$-comodule. 
Proof. Let $\mathcal{O}:=\mathcal{O}_{q}(G), \mathcal{O}_{n}:=\mathcal{O}_{q}(G / H)_{n}$, and set $\mathcal{O}^{\prime}:=\mathbb{k}(q) \otimes_{\mathbb{k}_{q}} \mathcal{O}, \mathcal{O}_{n}^{\prime}:=$ $\mathbb{k}(q) \otimes_{\mathbb{k}_{q}} \mathcal{O}_{n}$. Then $\mathcal{O} \otimes_{\mathbb{k}_{q}} \mathcal{O}$ naturally embeds into $\mathcal{O}^{\prime} \otimes_{\mathbb{k}(q)} \mathcal{O}^{\prime}$ because $\mathcal{O}$ and $\mathcal{O}_{n}^{\prime}$ are torsion free as $\mathbb{k}_{q}$-modules. Using this embedding, given any $\ell \in \mathcal{O}_{q}(G / H)_{n}$ we can always write $\Delta(\ell)=\sum_{i} g_{i}^{\prime} \otimes h_{i}^{\prime}$ for some $g_{i}^{\prime}, h_{i}^{\prime} \in \mathcal{O}^{\prime}$ such that the $g_{i}^{\prime \prime}$ 's are all linearly independent, and similarly for the $h_{i}^{\prime}$ 's. Then (taking a common denominator) there exists $c \in \mathbb{k}_{q}$ such that $\Delta(\ell)=c^{-1} \sum_{i} g_{i} \otimes h_{i}$, with the $g_{i}$ 's in $\mathcal{O}_{q}(G)$ being linearly independent and the $h_{i}$ 's in $\mathcal{O}_{q}(G)$, which are linearly independent too.

We shall now prove that

$$
\sum_{i} g_{i} \otimes \sum_{\left(h_{i}\right)}\left(h_{i}\right)_{(1)} \otimes \overline{\left(h_{i}\right)_{(2)}}=\sum_{i} g_{i} \otimes h_{i} \otimes \overline{d^{n}} .
$$

Indeed, the left-hand side of (3.1) is just the image of $c \ell$ via the map

$$
(\mathrm{id} \otimes((\mathrm{id} \otimes \pi) \circ \Delta)) \circ \Delta=(\mathrm{id} \otimes \mathrm{id} \otimes \pi) \circ(\mathrm{id} \otimes \Delta) \circ \Delta .
$$

By coassociativity of $\Delta$, the latter map coincides with $(\mathrm{id} \otimes \mathrm{id} \otimes \pi) \circ(\mathrm{id} \otimes \Delta) \circ \Delta=(\Delta \otimes \pi) \circ \Delta=(\Delta \otimes \mathrm{id}) \circ(\mathrm{id} \otimes \pi) \circ \Delta=(\Delta \otimes \mathrm{id}) \circ \Delta_{\pi}$, and now the last map applied to $c \ell$ gives

$$
(\Delta \otimes \mathrm{id})\left(\Delta_{\pi}(c \ell)\right) \stackrel{\circledast}{=}(\Delta \otimes \mathrm{id})\left(c \ell \otimes \overline{d^{n}}\right)=\sum_{i} g_{i} \otimes h_{i} \otimes \overline{d^{n}},
$$

where $\stackrel{\circledast}{=}$ follows from the assumption $\ell \in \mathcal{O}_{q}(G / H)_{n}$, which implies that $c \ell \in$ $\mathcal{O}_{q}(G / H)_{n}$ as well. This eventually gives the right-hand side of (3.1).

Now, because of the linear independence of the $h_{i}$ 's, the identity (3.1) implies that all the $h_{i}$ 's satisfy $\sum_{\left(h_{i}\right)}\left(h_{i}\right)_{(1)} \otimes \overline{\left(h_{i}\right)_{(2)}}=h_{i} \otimes \overline{d^{n}}$, which means exactly that $h_{i} \in \mathcal{O}_{q}(G / H)_{n}$ for every index $i$. Thus we have

$$
\Delta(c \ell)=\sum_{i} g_{i} \otimes h_{i}, \quad g_{i} \in \mathcal{O}_{q}(G), h_{i} \in \mathcal{O}_{q}(G / H)_{n},
$$

the $g_{i}$ 's resp. $h_{i}$ 's being linearly independent, and also

$$
\Delta(c \ell) \in c \mathcal{O}_{q}(G) \otimes \mathcal{O}_{q}(G) .
$$

These two conditions imply that $h_{i} \in \mathcal{O}_{q}(G / H)_{n} \cap c \mathcal{O}_{q}(G)=c \mathcal{O}_{q}(G / H)_{n}$ due to Remark 3.9. Therefore $\Delta(\ell) \in \mathcal{O}_{q}(G) \otimes \mathcal{O}_{q}(G / H)_{n}$. Finally, the claim for $\mathcal{O}_{q}(G / H)$ follows from that for the $\mathcal{O}_{q}(G / H)_{n}$ 's.

The above construction provides us with a reasonable candidate for a quantum analogue of $\mathcal{O}(G / H)$, namely the space of the $d$-semi-invariants $\mathcal{O}_{q}(G / H)$, which we proved has many important properties. Nevertheless, we still would like $\mathcal{O}_{q}(G / H)$ to verify three more key properties, namely: 
(a) $\mathcal{O}_{q}(G / H)$ is a subalgebra of $\mathcal{O}_{q}(G)$;

(b) $\mathcal{O}_{q}(G / H)$ is a graded object, its $n$-th (for all $n \in \mathbb{N}$ ) graded summand being $\mathcal{O}_{q}(G / H)_{n}$;

(c) the grading is compatible with all other structures, so $\mathcal{O}_{q}(G / H)$ is a graded $\mathcal{O}_{q}(G)$-comodule algebra (when $\mathcal{O}_{q}(G)$ is given the trivial grading).

Indeed, we are still missing these properties so far. In order to have them, an additional property must be required in addition to the pre-quantum section $d$ we started from. This is provided by the following result.

Proposition 3.11. Set $I_{q}(H):=\operatorname{Ker}(\pi)$, and let $d$ be a pre-quantum section on $G / H$. Then the following properties are equivalent:

(a) $\mathcal{O}_{q}(G / H)_{r} \cdot \mathcal{O}_{q}(G / H)_{s} \subseteq \mathcal{O}_{q}(G / H)_{r+s}$ for all $r, s \in \mathbb{N}$, hence, in particular, $\mathcal{O}_{q}(G / H)$ is a $\mathbb{k}_{q}$-subalgebra of $\mathcal{O}_{q}(G)$;

(b) $\overline{[d, f]}=\overline{0}$ in $\mathcal{O}_{q}(H)$ for all $f \in \mathcal{O}_{q}(G / H)$;

(c) $\left[d, \mathcal{O}_{q}(G / H)\right] \subseteq I_{q}(H)$.

Proof. (b) $\Longrightarrow$ (a): For any $r, s \in \mathbb{N}$ pick $f \in \mathcal{O}_{q}(G / H)_{r}, g \in \mathcal{O}_{q}(G / H)_{s}$. Then, by Proposition 3.10, for $\Delta(f)=\sum_{(f)} f_{(1)} \otimes f_{(2)}$ and $\Delta(g)=\sum_{(g)} g_{(1)} \otimes g_{(2)}$ we have $f_{(2)} \in \mathcal{O}_{q}(G / H)_{r}, g_{(2)} \in \mathcal{O}_{q}(G / H)_{s}$. This, along with assumption (b) to get the equality $\stackrel{\circledast}{=}$, yields the chain of identities

$$
\begin{aligned}
\Delta_{\pi}(f g) & =\sum_{(f),(g)} f_{(1)} g_{(1)} \otimes \overline{f_{(2)} g_{(2)}}=\sum_{(f),(g)} f_{(1)} g_{(1)} \otimes f_{(2)} \cdot \overline{g_{(2)}} \\
& =\sum_{(f),(g)}\left(f_{(1)} \otimes f_{(2)}\right) \cdot\left(g_{(1)} \otimes \overline{g_{(2)}}\right) \\
& =\left(\sum_{(f)} f_{(1)} \otimes f_{(2)}\right) \cdot\left(\sum_{(g)} g_{(1)} \otimes \overline{g_{(2)}}\right) \\
& =\left(\sum_{(f)} f_{(1)} \otimes f_{(2)}\right) \cdot\left(g \otimes \overline{d^{s}}\right)=\sum_{(f)} f_{(1)} g \otimes f_{(2)} \cdot \overline{d^{s}} \\
& =\sum_{(f)} f_{(1)} g \otimes \overline{f_{(2)} d^{s}} \stackrel{\otimes}{=} \sum_{(f)} f_{(1)} g \otimes \overline{d^{s} f_{(2)}} \\
& =\sum_{(f)} f_{(1)} g \otimes\left(d^{s} f_{(2)}\right) \cdot \overline{1}=\left(\left(1 \otimes d^{s}\right) \cdot \sum_{(f)} f_{(1)} \otimes f_{(2)}\right) \cdot(g \otimes \overline{1}) \\
& =\left(\left(1 \otimes d^{s}\right) \Delta(f)\right) \cdot(g \otimes \overline{1})=\left(\left(1 \otimes d^{s}\right)\left(f \otimes d^{r}+\varphi \otimes \eta\right)\right) \cdot(g \otimes \overline{1}) \\
& =\left(1 \otimes d^{s}\right) \cdot\left(f g \otimes \overline{d^{r}}+\varphi g \otimes \bar{\eta}\right)=f g \otimes \overline{d^{s+r}}=f g \otimes \pi\left(d^{s+r}\right)
\end{aligned}
$$

for some suitable $\varphi \in \mathcal{O}_{q}(G), \eta \in I_{q}(H)$, with notation $(x \otimes y) \cdot(a \otimes \bar{b}):=$ $(x a) \otimes(y \cdot \bar{b})$ referring to the action of $\mathcal{O}_{q}(G) \otimes \mathcal{O}_{q}(G)$ onto $\mathcal{O}_{q}(G) \otimes \mathcal{O}_{q}(H)$ 
induced by the action of $\mathcal{O}_{q}(G)$ onto $\mathcal{O}_{q}(H)$, via $\pi$, and onto itself, via left regular representation. So $f g$ is also $d$-semi-invariant of degree $r+s$.

(a) $\Longrightarrow$ (b): Assume that (a) holds. Then for $f \in \mathcal{O}_{q}(G / H)_{n}$ we have $d f$, $f d \in \mathcal{O}_{q}(G / H)_{n+1}$, and so $[d, f] \in \mathcal{O}_{q}(G / H)_{n+1}$. Then the identity

$$
\overline{[d, f]}=\epsilon([d, f]) \overline{d^{\partial(f)+1}}
$$

holds by Proposition 3.8. But clearly $\epsilon([d, f])=0$, hence $\overline{[d, f]}=\overline{0}$, that is, $[d, f] \in I_{q}(H)$. The outcome is $\left[d, \mathcal{O}_{q}(G / H)\right] \subseteq I_{q}(H)$.

(b) $\Longrightarrow$ (c): This is just a matter of rephrasing.

Definition 3.12. We call quantization of $t$, or quantum section (of the line bundle $\mathscr{L}$ ) on $G / H$, any pre-quantum section $d$ of $G / H$ (cf. Definition 3.5) which satisfies any of the equivalent conditions in Proposition 3.11.

The following result gives a criterion to detect quantum sections, and shows that for quantum subgroups they are just pre-quantum sections.

Proposition 3.13. (a) Let $d$ be a pre-quantum section on $G / H$. If $\left[d, I_{q}(H)\right] \subseteq$ $I_{q}(H)$, then $d$ is a quantum section.

(b) Let $\mathcal{O}_{q}(H)$ be a quantum subgroup. Then any pre-quantum section on $G / H$ is a quantum section.

Proof. (a) Pick any $f \in \mathcal{O}_{q}(G / H)_{r}, g \in \mathcal{O}_{q}(G / H)_{s}$. Definition 3.7 gives

$$
\begin{array}{cl}
\Delta(f)=f \otimes d^{r}+f_{1} \otimes \phi_{1}, & f_{1} \in \mathcal{O}_{q}(G), \phi_{1} \in I_{q}(H), \\
\Delta(g)=g \otimes d^{S}+g_{1} \otimes \gamma_{1}, & g_{1} \in \mathcal{O}_{q}(G), \gamma_{1} \in I_{q}(H) .
\end{array}
$$

Therefore, for the product $f g$ we have

$$
\begin{aligned}
\Delta(f g)=\Delta(f) \Delta(g) & =\left(f \otimes d^{r}+f_{1} \otimes \phi_{1}\right)\left(g \otimes d^{s}+g_{1} \otimes \gamma_{1}\right) \\
& =f g \otimes d^{r+s}+f g_{1} \otimes d^{r} \gamma_{1}+f_{1} g \otimes \phi_{1} d^{s}+f_{1} g_{1} \otimes \phi_{1} \gamma_{1} .
\end{aligned}
$$

Now $d^{r} \gamma_{1}, \phi_{1} \gamma_{1} \in I_{q}(H)$ because $I_{q}(H)$ is a (left) $\mathcal{O}_{q}(G)$-submodule, and

$$
\phi_{1} d^{s}=d^{s} \phi_{1}+\left[\phi_{1}, d^{s}\right] \in\left(I_{q}(H)+I_{q}(H)\right)=I_{q}(H)
$$

because, in addition, $\left[d, I_{q}(H)\right] \subseteq I_{q}(H)$ by assumption (d). Thus

$$
\Delta(f g) \in\left(f g \otimes d^{r+s}+\mathcal{O}_{q}(G) \otimes I_{q}(H)\right),
$$

which means exactly that $f g \in \mathcal{O}_{q}(G / H)_{r+s}$, again by Definition 3.7. Thus condition (a) of Proposition 3.11 holds, hence we conclude by Definition 3.12.

(b) If $\mathcal{O}_{q}(H)$ is a quantum subgroup, then $I_{q}(H)$ is a two-sided ideal. Therefore, $\left[d, I_{q}(H)\right] \subseteq I_{q}(H)$, hence by (a) we get the claim. 
The following result records yet another feature of quantum sections.

Lemma 3.14. Let $d$ be a quantum section on $G / H$. Then $d^{n} \in \mathcal{O}_{q}(G / H)_{n}$, and $\overline{d^{n}}$ is group-like in $\mathcal{O}_{q}(H)$ for all $n \in \mathbb{N}$. Moreover, $\operatorname{span}_{\mathbb{k}_{q}}\left(\left\{{\overline{d^{n}}}_{\}_{n \in \mathbb{N}}}\right)\right.$ is a $\mathbb{k}_{q^{-s u b-}}$ coalgebra of $\mathcal{O}_{q}(H)$ and

$$
\operatorname{span}_{\mathbb{k}_{q}}\left(\left\{\overline{d^{n}}\right\}_{n \in \mathbb{N}}\right)=\bigoplus_{n \in \mathbb{N}} \mathbb{k}_{q} \overline{d^{n}}
$$

Proof. By Definition 3.12 and condition (a) of Proposition 3.11 we have that $d^{n} \in$ $\mathcal{O}_{q}(G / H)_{n}$ for all $n \in \mathbb{N}$. This means that $\Delta\left(d^{n}\right) \in\left(d^{n} \otimes d^{n}+\mathcal{O}_{q}(G) \otimes I_{q}(H)\right)$, whence, as $\pi: \mathcal{O}_{q}(G) \rightarrow \mathcal{O}_{q}(H)$ is a coalgebra morphism, we obtain that

$$
\Delta\left(\overline{d^{n}}\right)=\Delta\left(\pi\left(d^{n}\right)\right)=(\pi \otimes \pi)\left(\Delta\left(d^{n}\right)\right)=\pi\left(d^{n}\right) \otimes \pi\left(d^{n}\right)=\overline{d^{n}} \otimes \overline{d^{n}} .
$$

Thus the $\overline{d^{n}}$, s are group-like and different from other since so are the $t^{n}=\left.d^{n}\right|_{q=1}$. Finally, this implies that $\operatorname{span}_{\mathbb{k}_{q}}\left(\left\{\bar{d}^{n}\right\}_{n \in \mathbb{N}}\right)$ is a $\mathbb{k}_{q}$-subcoalgebra of $\mathcal{O}_{q}(H)$ and that the $\overline{d^{n}}$ 's are linearly independent, which gives $\operatorname{span}_{\mathbb{k}_{q}}\left(\left\{\overline{d^{n}}\right\}_{n \in \mathbb{N}}\right)=\bigoplus_{n \in \mathbb{N}} \mathbb{k}_{q} \overline{d^{n}}$.

Collecting all the previous results, we can now show that semi-invariants built out of a quantum section satisfy all properties we look for.

Theorem 3.15. Let $d$ be a quantum section on $G / H$. Then:

(a) $\mathcal{O}_{q}(G / H)$ is a graded $\mathbb{k}_{q}$-module, its $n$-th graded summand $(n \in \mathbb{N}$ ) being $\mathcal{O}_{q}(G / H)_{n}$.

(b) $\mathcal{O}_{q}(G / H)$ is a subalgebra of $\mathcal{O}_{q}(G)$.

(c) The grading in (a) is compatible with all other structures of $\mathcal{O}_{q}(G / H)$, so that $\mathcal{O}_{q}(G / H)$ is a graded $\mathcal{O}_{q}(G)$-comodule algebra, where we take on $\mathcal{O}_{q}(G)$ the trivial grading.

(d) For every $c \in \mathbb{k}_{q}$, we have $\mathcal{O}_{q}(G / H) \cap c \mathcal{O}_{q}(G)=c \mathcal{O}_{q}(G / H)$. In particular, $\mathcal{O}_{q}(G / H) \cap(q-1) \mathcal{O}_{q}(G)=(q-1) \mathcal{O}_{q}(G / H)$.

Proof. (a) We must simply prove that the sum $\sum_{n \in \mathbb{N}} \mathcal{O}_{q}(G / H)_{n}$ is direct so that $\mathcal{O}_{q}(G / H):=\sum_{n \in \mathbb{N}} \mathcal{O}_{q}(G / H)_{n}=\bigoplus_{n \in \mathbb{N}} \mathcal{O}_{q}(G / H)_{n}$. But this is an easy consequence of Lemma 3.14.

Indeed, let $\sum_{n \in \mathbb{N}} c_{n} f_{n}=0$ a linear dependence relation, with $f_{n} \in \mathcal{O}_{q}(G / H)_{n}$ and $c_{n} \in \mathbb{k}_{q}$ (almost all zero) for every $n \in \mathbb{N}$. Applying $\Delta_{\pi}$ to this relation we get $\sum_{n \in \mathbb{N}} c_{n} f_{n} \otimes \overline{d^{n}}=0$. But by Lemma 3.14 the $\overline{d^{n}}$,s are linearly independent; thus $c_{n}=0$ for all $n$.

(b) This follows directly from Definition 3.12 and Proposition 3.11. 
(c) This follows (again) from Definition 3.12 and Proposition 3.11 as well as from Proposition 3.10 and the Hopf algebra axioms.

(d) This easily follows from the identity $\mathcal{O}_{q}(G / H)=\bigoplus_{n \in \mathbb{N}} \mathcal{O}_{q}(G / H)_{n}$ given by claim (a), and from Remark 3.9.

Corollary 3.16. Let $d$ be a quantum section on $G / H$ (in the sense of Definition 3.12). Then the restriction of $\pi: \mathcal{O}_{q}(G) \rightarrow \mathcal{O}_{q}(H)$ yields an epimorphism of graded $\mathcal{O}_{q}(G)$-module coalgebras:

$$
\pi^{\prime}: \mathcal{O}_{q}(G / H) \rightarrow \operatorname{span}_{\mathbb{k}_{q}}\left(\left\{\overline{d^{n}}\right\}_{n \in \mathbb{N}}\right)=\bigoplus_{n \in \mathbb{N}} \mathbb{k}_{q} \overline{d^{n}}
$$

Proof. By Lemma 3.8 we know that $\pi^{\prime}$ is a well-defined epimorphism of graded $\mathbb{k}_{q}$-modules. The rest follows from $\mathcal{O}_{q}(G / H)$ being a subalgebra of $\mathcal{O}_{q}(G)$, and $\pi$ being a morphism of $\mathcal{O}_{q}(G)$-module coalgebras.

The last aspect to take into account is the behavior of $\mathcal{O}_{q}(G / H)$ under specialization at $q=1$. The last part of claim (d) of Theorem 3.15 ensures that such specialization is consistent with that of $\mathcal{O}_{q}(G)$ : in other words, the embedding $\mathcal{O}_{q}(G / H) \hookrightarrow \mathcal{O}_{q}(G)$ gets down under specialization to an embedding $\mathcal{O}_{1}(G / H):=$ $\mathcal{O}_{q}(G / H) /(q-1) \mathcal{O}_{q}(G / H) \hookrightarrow \mathcal{O}(G)$. The next result tells us something about the specialized space $\mathcal{O}_{1}(G / H)$ itself.

Proposition 3.17. $\mathcal{O}_{1}(G / H):=\mathcal{O}_{q}(G / H) /(q-1) \mathcal{O}_{q}(G / H)$ is a graded Poisson subalgebra of $\mathcal{O}(G / H)=\bigoplus_{n \in \mathbb{N}} \mathcal{O}(G / H)_{n}$ and a graded left coideal of $\mathcal{O}(G)$, with $\mathcal{O}_{1}(G / H)_{n}:=\mathcal{O}_{q}(G / H)_{n} /(q-1) \mathcal{O}_{q}(G / H)_{n}$ as $n$-th graded summand $(n \in \mathbb{N})$. In particular, it is a left $\mathcal{O}(G)$-comodule algebra.

Proof. For all $n \in \mathbb{N}$, the restriction to $\mathcal{O}_{q}(G / H)_{n}$ of the specialization map

$$
p_{1}: \mathcal{O}_{q}(G) \rightarrow \mathcal{O}_{q}(G) /(q-1) \mathcal{O}_{q}(G) \cong \mathcal{O}(G)
$$

has kernel $\mathcal{O}_{q}(G / H)_{n} \cap(q-1) \mathcal{O}_{q}(G)=(q-1) \mathcal{O}_{q}(G / H)_{n}$ by Remark 3.9. This in turn ensures also that the restriction of $p_{1}$ to $\mathcal{O}_{q}(G / H)=\bigoplus_{n \in \mathbb{N}} \mathcal{O}_{q}(G / H)_{n}$ has kernel $\bigoplus_{n \in \mathbb{N}}(q-1) \mathcal{O}_{q}(G / H)_{n}$, so its image is $\mathcal{O}_{q}(G / H) /(q-1) \mathcal{O}_{q}(G / H)$, i.e., just the specialization of $\mathcal{O}_{q}(G / H)$. So

$$
\mathcal{O}_{1}(G / H):=\mathcal{O}_{q}(G / H) /(q-1) \mathcal{O}_{q}(G / H)=p_{1}\left(\mathcal{O}_{q}(G / H)\right)
$$

where the right-hand side is a subalgebra of $p_{1}\left(\mathcal{O}_{q}(G)\right)=\mathcal{O}(G)$. Moreover, we have also that the specialization maps preserves the grading, namely

$$
p_{1}\left(\mathcal{O}_{q}(G / H)\right)=p_{1}\left(\bigoplus_{n \in \mathbb{N}} \mathcal{O}_{q}(G / H)_{n}\right)=\bigoplus_{n \in \mathbb{N}} p_{1}\left(\mathcal{O}_{q}(G / H)_{n}\right)
$$


so that $\mathcal{O}_{1}(G / H)$ is graded, with $n$-th graded summand

$$
p_{1}\left(\mathcal{O}_{q}(G / H)_{n}\right)=\mathcal{O}_{q}(G / H)_{n} /(q-1) \mathcal{O}_{q}(G / H)_{n}=: \mathcal{O}_{1}(G / H)_{n} .
$$

Now Theorem 3.15 implies at once that $\mathcal{O}_{1}(G / H)$ is a graded subalgebra left coideal inside $p_{1}\left(\mathcal{O}_{q}(G)\right)=\mathcal{O}(G)$, hence a graded (left) $\mathcal{O}(G)$-comodule algebra. In addition, the identity

$$
\mathcal{O}_{q}(G / H) \cap(q-1) \mathcal{O}_{q}(G)=(q-1) \mathcal{O}_{q}(G / H)
$$

implies also that the Poisson bracket defined in $\mathcal{O}(G / H)$ starting from its quantization $\mathcal{O}_{q}(G / H)$ - see Remark 3.2 - coincides with the restriction to $\mathcal{O}(G / H)$ of the Poisson bracket similarly induced on $\mathcal{O}(G)$ from $\mathcal{O}_{q}(G)$. Therefore, $\mathcal{O}_{1}(G / H)$ is also a Poisson subalgebra of $\mathcal{O}(G)$.

We are only left to prove that the embedding of $\mathcal{O}_{1}(G / H)$ into $\mathcal{O}(G)$ maps $\mathcal{O}_{1}(G / H)$ into $\mathcal{O}(G / H)$ respecting the grading on either side, that is

$$
\mathcal{O}_{1}(G / H)_{n} \subseteq \mathcal{O}(G / H)_{n} \text { for all } n
$$

Now the left-hand side of (3.2) is $\mathcal{O}_{q}(G / H)_{n} /(q-1) \mathcal{O}_{q}(G / H)_{n}$, with

$$
\mathcal{O}_{q}(G / H)_{n}:=\left\{\ell \in \mathcal{O}_{q}(G) \mid \Delta_{\pi}(\ell)=\ell \otimes \pi\left(d^{n}\right)\right\}
$$

(cf. Definition 3.7), while the right-hand side, by (2.4), is

$$
\mathcal{O}(G / H)_{n}=\left\{f \in \mathcal{O}(G) \mid \Delta_{\pi}(f)=f \otimes \pi\left(t^{n}\right)\right\}
$$

But all specialization maps commute with the coproducts $\Delta$ and with the (quantum and classical) maps $\pi$, and the specialization of each $d^{n}$ is nothing but $t^{n}$. Therefore, we conclude that (3.2) holds.

Finally, we can define our "quantum projective homogeneous spaces".

Definition 3.18. Let $G$ be an algebraic Poisson group, $H$ a coisotropic subgroup as in $\S 2.2$, and let $\mathcal{O}_{q}(G), \mathcal{O}_{q}(H)$ and $\pi: \mathcal{O}_{q}(G) \rightarrow \mathcal{O}_{q}(H)$ be given (cf. Definitions 3.1, 3.3). Let $d$ be a quantum section on $G / H$ (see Definition 3.12, Proposition 2.9), in particular

(a) $\Delta_{\pi}(d)=d \otimes \pi(d)$;

(b) $d \equiv t \bmod (q-1)$, where $t$ is a non-zero section of the very ample line bundle on $G / H$ giving the embedding into some projective space.

We say that

$$
O_{q}(G / H):=\bigoplus_{n \in \mathbb{N}} \mathcal{O}_{q}(G / H)_{n}
$$


with

$$
\begin{aligned}
\mathcal{O}_{q}(G / H)_{n}: & =\left\{\ell \in \mathcal{O}_{q}(G) \mid \Delta_{\pi}(\ell)=\ell \otimes \pi\left(d^{n}\right)\right\} \\
& =\left\{\ell \in \mathcal{O}_{q}(G) \mid \Delta(\ell) \in\left(\ell \otimes d^{n}+\mathcal{O}_{q}(G) \otimes I_{q}(H)\right)\right\}
\end{aligned}
$$

is a quantization of $\mathcal{O}(G / H)$ if

$$
\mathcal{O}_{q}(G / H) /(q-1) \mathcal{O}_{q}(G / H) \cong \mathcal{O}(G / H)
$$

as graded $\mathcal{O}(G)$-module algebras and as Poisson algebras over $\mathbb{k}$. We will then refer to $\mathcal{O}_{q}(G / H)$ as quantum projective homogeneous space.

In particular, we have seen that any such $\mathcal{O}_{q}(G / H)$ has the following properties (Theorem 3.15):

(I) it is a graded subalgebra of $\mathcal{O}_{q}(G)$;

(II) it is a left coideal of $\mathcal{O}_{q}(G)$, hence a left $\mathcal{O}_{q}(G)$-comodule via

$$
\left.\Delta\right|_{\mathcal{O}_{q}(G / H)}: \mathcal{O}_{q}(G / H) \rightarrow \mathcal{O}_{q}(G) \otimes \mathcal{O}_{q}(G / H) .
$$

Remark 3.19. As a matter of fact, the only additional property required in Definition 3.18 is that the embedding of $\mathcal{O}_{q}(G / H) /(q-1) \mathcal{O}_{q}(G / H)$ into $\mathcal{O}(G / H)$ provided by Proposition 3.17 be onto. But actually, as both these are graded algebras, and $\mathcal{O}(G / H)$ is generated in degree one, this is equivalent to requiring (only) the embedding

$$
\mathcal{O}_{q}(G / H)_{1} /(q-1) \mathcal{O}_{q}(G / H)_{1} \hookrightarrow \mathcal{O}(G / H)_{1}
$$

be onto.

This requirement might be seen as the quantum analogue of the requirement - at the semiclassical level, see Section 2.2 - of having enough global sections of the line bundle $\mathscr{L}$ on $G / H$ in order to have an embedding of $G / H$ into $\mathbb{P}^{N}$.

In Section 5 we show that quantum Grassmannians and quantum generalized flag varieties are examples of quantum projective homogeneous space.

\section{The quantum duality principle (QDP)}

4.1. The QDP philosophy. The quantum duality principle (QDP) is a two-fold recipe which allows to obtain a quantum group dual, in an appropriate sense, to a given one.

In [5] Ciccoli and Gavarini extended this principle to quantum formal homogeneous spaces. Their result goes as follows. 
Given a Poisson group $G$, we consider pairs $(H, G / H)$ where $H$ is a coisotropic subgroup of $G$ and $G / H$ is the corresponding homogeneous space. At a local level, i.e., in the setup of formal geometry, any such pair can be described in algebraic terms by any one of the following four objects,

$$
U(\mathfrak{h}), \quad U(\mathfrak{g}) \mathfrak{h}, \quad I(H), \quad \mathcal{O}(G / H)
$$

where hereafter $\mathfrak{h}:=\operatorname{Lie}(H)$ and $\mathfrak{g}:=\operatorname{Lie}(G)$, and the notation is standard except for $\mathcal{O}(G / H)$ which here denotes the algebra of regular functions on the formal algebraic variety $G / H$. The main result in [5] is a four-fold functorial recipe which, from a quantization of each object in (4.1), constructs a quantization of one of the four objects of the similar quadruple which describes the "dual" pair $\left(H^{\perp}, G^{*} / H^{\perp}\right)$, where "dual" refers to Poisson duality.

If we try to do the same at a global level (cf. [6]), i.e., not restricting ourselves to the framework of formal geometry, then something changes when handling the algebra $\mathcal{O}(G / H)$. Namely, the latter is meaningful - that is, it permits to get back the pair $(H, G / H)-$ only if $G / H$ is a quasi-affine variety. This is the case, in particular, if $G / H$ is affine, but it is not so if $G / H$ is projective. Therefore, in the latter case one describes $G / H$ taking instead of the algebra of regular functions the algebra of (algebraic) sections of a line bundle on $G / H$ realizing an embedding in a projective space, i.e., what is denoted $\mathcal{O}(G / H)$ in Section 2. Once this is settled, one can consider a quantization $\mathcal{O}_{q}(G / H)$ and try to cook up a suitable analogue of the recipe of [5], [6].

With this program in mind, we want to associate to any quantum homogeneous $G$-space $\mathcal{O}_{q}(G / H)$ - in the sense of Definition 3.18 - a (local) quantization $U_{q}\left(\mathfrak{h}^{\perp}\right)$ of the dual $G^{*}$-space (actually, of the dual coisotropic subgroup), right in the spirit of the QDP.

Warning. In order to make our statements simpler, from now on we take as ground ring the local ring

$$
\mathbb{k}_{q}^{\prime}:=\left(\mathbb{k}_{q}\right)_{(q-1)}=\text { localization of } \mathbb{k}_{q} \text { at the ideal generated by }(q-1) .
$$

Therefore, hereafter we shall tacitly extend scalars from $\mathbb{k}_{q}$ to $\mathbb{k}_{q}^{\prime}$ for all $\mathbb{k}_{q}$-modules and $\mathbb{k}_{q}$-algebras we have considered so far, with no further mention.

Let $G$ be an affine algebraic group and $H$ a closed coisotropic parabolic subgroup, i.e., $G / H$ is a projective homogeneous space. Let $\mathcal{O}_{q}(G), \mathcal{O}_{q}(H)$ and $\mathcal{O}_{q}(G / H)$ be quantum deformations of $\mathcal{O}(G), \mathcal{O}(H)$ and $\mathcal{O}(G / H)$ as defined in Section 3. In particular, $\mathcal{O}_{q}(G / H)$ is built out of a specific quantum section $d$ on $G / H$. Also, $\pi(d)=\bar{d} \in \mathcal{O}_{q}(H)$ is (non-zero) group-like, hence $\epsilon(d)=\epsilon(\bar{d})=1$, and $d$ specializes to $\left.d\right|_{q=1}=t \in \mathcal{O}(G / H) \subseteq \mathcal{O}(G)$.

In addition, we make the following assumption: $d$ is an Ore element in the algebra $\mathcal{O}_{q}(G)$. 
This property will allow us to enlarge the algebras $\mathcal{O}_{q}(G)$ and $\mathcal{O}_{q}(G / H)$ by the formal inverse $d^{-1}$. Geometrically, it corresponds to asking - besides a quantization of $\mathcal{O}(G / H)$ - for a quantization of a Zariski neighbourhood of the identity element; more precisely, it means that we have a quantization of the function algebra $\mathcal{O}\left(X_{t}\right)$ of the affine variety $X_{t}$, the complement in $G / H$ of the divisor defined by the function $t=\left.d\right|_{q=1}$. This property is satisfied in the examples of the Grassmannian and the flag varieties (cf. Section 5), with a suitable choice of $d$.

Let us define

$$
\mathcal{O}_{q}^{\text {loc }}(G / H):=\mathcal{O}_{q}(G / H)\left[d^{-1}\right]_{\text {proj }} \subseteq \mathcal{O}_{q}(G)\left[d^{-1}\right]
$$

where the localization is a projective localization, i.e., we take the elements of degree zero in the ring $\mathcal{O}_{q}(G / H)\left[d^{-1}\right]$, where $d^{-1}$ is given degree -1 .

4.2. The QDP functor. We now recall the definition of the functor $\mathcal{O}_{q}(G) \mapsto$ $\mathcal{O}_{q}(G)^{\vee}$, which sends quantized function algebras (of Poisson groups) to quantized universal algebras (of Lie bialgebras). More precisely, $\mathcal{O}_{q}(G)^{\vee}$ is a quantization of $U\left(\mathrm{~g}^{*}\right)$, where $\mathrm{g}^{*}$ is the Lie bialgebra dual to $\mathrm{g}$. For more details and proofs, we refer the reader to [14], Theorem 2.2 and Theorem 4.7.

Remark. The overall assumption in [14] for $G$ is to be connected; nevertheless, this condition is not needed in the proof of Theorem 4.7 therein.

Definition 4.1. Let $J_{G}:=\operatorname{Ker}\left(\epsilon: \mathcal{O}_{q}(G) \rightarrow \mathbb{k}_{q}^{\prime}\right)$ be the augmentation ideal of $\mathcal{O}_{q}(G)$. Also, let $I_{G}:=J_{G}+(q-1) \mathcal{O}_{q}(G)$. We define

$$
\mathcal{O}_{q}(G)^{\vee}:=\sum_{n \geq 0}(q-1)^{-n} I_{G}^{n}=\sum_{n \geq 0}\left((q-1)^{-1} I_{G}\right)^{n}=\bigcup_{n \geq 0}\left((q-1)^{-1} I_{G}\right)^{n} .
$$

This is a well-defined $\mathbb{k}_{q}^{\prime}$-subalgebra of $\mathbb{k}(q) \otimes_{\mathbb{k}_{q}^{\prime}} \mathcal{O}_{q}(G)$. Notice also that

$$
\mathcal{O}_{q}(G)^{\vee}=\sum_{n \geq 0}(q-1)^{-n} J_{G}^{n}=\sum_{n \geq 0}\left((q-1)^{-1} J_{G}\right)^{n} .
$$

The results of [14] - in particular, Theorem 4.7 therein - tell us that $\mathcal{O}_{q}(G)^{\vee}$ is a quantization of $U\left(\mathrm{~g}^{*}\right)$, that is, $\mathcal{O}_{q}(G)^{\vee} /(q-1) \mathcal{O}_{q}(G)^{\vee} \cong U\left(\mathrm{~g}^{*}\right)$ as co-Poisson Hopf algebras. Our idea is to somehow "restrict to $\mathcal{O}_{q}(G / H)$ " the definition of $\mathcal{O}_{q}(G)^{\vee}$, so as to define $\mathcal{O}_{q}(G / H)^{\vee}$. To begin with, let

$$
J_{G / H}:=\operatorname{Ker}\left(\left.\epsilon\right|_{\mathcal{O}_{q}(G / H)}\right) \text {. }
$$

Notice that $\epsilon$ extends uniquely to $\mathcal{O}_{q}(G)\left[d^{-1}\right]$, so we can also define

$$
J_{G / H}^{\mathrm{loc}}:=\operatorname{Ker}\left(\left.\epsilon\right|_{\mathcal{O}_{q}^{\mathrm{loc}}(G / H)}\right) \text {. }
$$


Definition 4.2. We define

$$
\mathcal{O}_{q}(G / H)^{\vee}:=\sum_{n \geq 0}(q-1)^{-n}\left(J_{G / H}^{\mathrm{loc}}\right)^{n}=\sum_{n \geq 0}\left((q-1)^{-1} J_{G / H}^{\mathrm{loc}}\right)^{n},
$$

the unital $\mathbb{k}_{q}^{\prime}$-subalgebra of $\mathbb{k}(q) \otimes_{\mathbb{k}_{q}^{\prime}} \mathcal{O}_{q}^{\text {loc }}(G / H)$ generated by $(q-1)^{-1} J_{G / H}^{\text {loc }}$, or what amounts to the same - by $(q-1)^{-1} I_{G / H}^{\text {loc }}$, where by definition we set $I_{G / H}^{\text {loc }}:=$ $J_{G / H}^{\text {loc }}+(q-1) \mathcal{O}_{q}^{\text {loc }}(G / H)$.

Indeed, one can check that the construction $\mathcal{O}_{q}(G / H) \mapsto \mathcal{O}_{q}(G / H)^{\vee}$ is functorial in a natural sense (see Remark 4.12 later on).

In order to study $\mathcal{O}_{q}(G / H)^{\vee}$, we need a rather explicit description of it. In turn, this requires a description of $\mathcal{O}_{q}(G)^{\vee}$, which we take from [14].

Let $J_{1}$ be the augmentation ideal of $\mathcal{O}(G)$, namely

$$
J_{1}:=\operatorname{Ker}(\epsilon: \mathcal{O}(G) \longrightarrow \mathbb{k})=J_{G} \bmod (q-1) \mathcal{O}_{q}(G)
$$

so that $J_{1} / J_{1}^{2}=\mathrm{g}^{*}$, the cotangent Lie bialgebra of $G$. Let $\left\{y_{1}, \ldots, y_{n}\right\}$ be a subset of $J_{1}$ whose image in the local ring of $G$ at $e$ (the unit element of the group $G$ ) is a local system of parameters; in particular, $n=\operatorname{dim}(G)$. Define $\left\{j_{1}, \ldots, j_{n}\right\}$ as a pull-back of $\left\{y_{1}, \ldots, y_{n}\right\}$ to $J_{G}$.

Theorem 4.3 (see [14], Lemma 4.1). (a) The set of ordered monomials $\left\{\prod_{s=1}^{n} j_{s}^{e_{s}} \mid \underline{e}=\left(e_{1}, \ldots, e_{n}\right) \in \mathbb{N}^{n}\right\}$ is a $\mathbb{k}_{q}^{\prime}$-pseudobasis (or topological basis) of $\widetilde{\mathcal{O}_{q}}(G)$, the $I_{G}$-adic completion of $\mathcal{O}_{q}(G)$. In other words, each element of $\widetilde{\mathcal{O}_{q}}(G)$ has a unique expansion as a formal infinite $\mathbb{k}_{q}^{\prime}$-linear combination of the $j \underline{e}$ 's. In particular, $\widetilde{\mathcal{O}_{q}}(G)$ is generated - as a topological $\mathbb{k}_{q}^{\prime}$-algebra - by $\left\{j_{1}, \ldots, j_{n}\right\}$.

(b) The $(q-1)$-adic completion $\widehat{\mathcal{O}_{q}}(G)^{\vee}$ of $\mathcal{O}_{q}(G)^{\vee}$ admits the set of ordered monomials $\left\{(q-1)^{-\left(e_{1}+\cdots+e_{n}\right)} \prod_{s=1}^{n} j_{s}^{e_{s}} \mid\left(e_{1}, \ldots, e_{n}\right) \in \mathbb{N}^{n}\right\}$ as a $\mathbb{k}_{q}^{\prime}$-pseudobasis. In particular, it follows that $\widehat{\mathcal{O}_{q}}(G)^{\vee}$ is generated - as a topological $\mathbb{k}_{q}^{\prime}$-algebra - by $\left\{j_{s}^{\vee}:=(q-1)^{-1} j_{s} \mid s=1, \ldots, n\right\}$.

The description of $\mathcal{O}_{q}(G / H)^{\vee}$ goes much along the same lines.

Recall that $t \in \mathcal{O}(G / H)$ is the specialization of $d \in \mathcal{O}_{q}(G / H)$. We then consider $X_{t}$, the open subvariety of $G / H$ where $t \neq 0$. On this variety, choose functions $l_{1}, \ldots, l_{n-h}$, where $h=\operatorname{dim}(H)$, such that the set

$$
\left\{x_{s}:=l_{s} \bmod (q-1) \mathcal{O}_{q}(G / H) \mid s=1, \ldots, n-h\right\}
$$

yields, in the localization of $\mathcal{O}\left(X_{t}\right)$ at $e H \in X_{t}(\subseteq G / H)$, a local system of parameters at $e H$. 
Theorem 4.4. (a) The set of ordered monomials

$$
\left\{\prod_{s=1}^{n-h} l_{s}^{e_{s}} \mid\left(e_{1}, \ldots, e_{n-h}\right) \in \mathbb{N}^{n-h}\right\}
$$

is a $\mathbb{k}_{q}^{\prime}$-pseudobasis of $\widetilde{\mathcal{O}_{q}^{\text {loc }}}(G / H)$, the latter being the $I_{G / H}^{\text {loc }}$-adic completion of $\mathcal{O}_{q}^{\text {loc }}(G / H)$ where $I_{G / H}^{\text {loc }}:=J_{G / H}^{\text {loc }}+(q-1) \mathcal{O}_{q}^{\text {loc }}(G / H)$.

In particular, $\widetilde{\mathcal{O}_{q}^{\text {loc }}}(G / H)$ is (topologically) generated by $\left\{l_{1}, \ldots, l_{n-h}\right\}$.

(b) The $(q-1)$-adic completion $\widehat{\mathcal{O}_{q}}(G / H)^{\vee}$ of $\mathcal{O}_{q}(G / H)^{\vee}$ admits as a $\mathbb{k}_{q}^{\prime}$-pseudobasis the set of ordered monomials

$$
\left\{(q-1)^{-\left(e_{1}+\cdots+e_{n-h}\right)} \prod_{s=1}^{n-h} l_{s}^{e_{s}} \mid\left(e_{1}, \ldots, e_{n-h}\right) \in \mathbb{N}^{n-h}\right\} .
$$

In particular, $\widehat{\mathcal{O}_{q}}(G / H)^{\vee}$ is (topologically) generated by the set

$$
\left\{l_{s}^{\vee}:=(q-1)^{-1} l_{s} \mid s=1, \ldots, n-h\right\} .
$$

Proof. The argument follows the proof of Theorem 4.9 in [15]. This theorem deals with the general setting of a quantization $\mathcal{O}_{q}(V)$ of any Poisson affine variety $V$ with a distinguished point on it, given by a character $\chi$ of $\mathcal{O}_{q}(V)$, the kernel of $\chi$ playing the role of $J_{G}$ above. Here we apply all this to $V=X_{t}(\subseteq G / H)$ with $\left.\epsilon\right|_{\mathcal{O}\left(X_{t}\right)}$ as character on $\mathcal{O}\left(X_{t}\right)=\mathcal{O}_{q}^{\text {loc }}(G / H)$.

The next lemma plays a crucial role in the construction of the QDP.

Lemma 4.5. The quantum section $d \in \mathcal{O}_{q}(G / H)$ enjoys the following properties:

(a) $d$ is invertible in $\widetilde{\mathcal{O}_{q}^{\text {loc }}}(G / H)$, with $d^{-1}=\sum_{k=0}^{+\infty}(1-d)^{k}$;

(b) $d$ is invertible in $\widehat{\mathcal{O}_{q}}(G / H)^{\vee}$, with $d^{-1}=\sum_{k=0}^{+\infty}(q-1)^{k}\left(\frac{(1-d)}{(q-1)}\right)^{k}$.

Proof. Observe that $\epsilon(d)=1$ implies that

$$
d=1-(1-d) \in\left(1+J_{G / H}^{\mathrm{loc}}\right) \subseteq\left(1+I_{G / H}^{\mathrm{loc}}\right)
$$

this gives the invertibility in $\widetilde{\mathcal{O}_{q}^{\text {loc }}}(G / H)$.

Similarly since $J_{G / H}^{\text {loc }} \subseteq(q-1) \mathcal{O}_{q}(G / H)^{\vee}$, the identity

$$
d=1+(d-1) \in\left(1+J_{G / H}^{\mathrm{loc}}\right) \subseteq\left(1+(q-1) \mathcal{O}_{q}(G / H)^{\vee}\right)
$$

also ensures that $d$ is invertible in $\widehat{\mathcal{O}_{q}}(G / H)^{\vee}$.

In both cases, the explicit formula for $d^{-1}$ follows by taking the limit of the geometric series, namely $(1-x)^{-1}=\sum_{k=0}^{+\infty} x^{k}$, applied to $x=1-d$. 
Proposition 4.6. There are natural embeddings

$$
\widetilde{\mathcal{O}_{q}}(G / H) \hookrightarrow \widetilde{\mathcal{O}_{q}}(G), \quad \widehat{\mathcal{O}_{q}}(G / H) \hookrightarrow \widehat{\mathcal{O}_{q}}(G),
$$

which both are extensions of the embedding $\mathcal{O}_{q}(G / H) \hookrightarrow \mathcal{O}_{q}(G)$. Moreover, via these embeddings the pseudobases for the (topological) algebras on $G / H$ identify with subsets of the corresponding ones for the (topological) algebras on $G$.

Proof. By construction, we have $l_{k}=j_{s} / d^{c_{s}}$ for some $j_{s} \in J_{G / H}, c_{s} \in \mathbb{N}$ $(s=1, \ldots, n-h)$. Since $d$ is invertible (in both cases), the previous analysis tells us that we can replace the $l_{k}$ 's with the $j_{k}$ 's $(k=1, \ldots, n-h)$ in the descriptions of $\widetilde{\mathcal{O}_{q}}(G / H)^{\vee}$ and $\widehat{\mathcal{O}_{q}}(G / H)^{\vee}$ given above (i.e., in the $\mathbb{k}_{q}^{\prime}$-pseudobases and as topological generators). But then, since

$$
\left\{j_{s} \mid s=1, \ldots, n-h\right\} \subseteq J_{G / H} \subseteq \mathcal{O}_{q}(G / H) \subseteq \mathcal{O}_{q}(G),
$$

we can always complete $\left\{j_{s} \mid s=1, \ldots, n-h\right\}$ to a set $\left\{j_{r} \mid r=1, \ldots, n\right\}$ such that $\left\{y_{r}:=j_{r} \bmod (q-1) \mathcal{O}_{q}(G) \mid r=1, \ldots, n\right\}$ yield, in the localization of $\mathcal{O}(G)$ at $e \in G$, a local system of parameters at $e$. Thus using the latter we can describe $\widetilde{\mathcal{O}_{q}}(G)$ and $\widehat{\mathcal{O}_{q}}(G)$ as explained above.

From now on we shall use these embeddings to identify $\widetilde{\mathcal{O}_{q}}(G / H)$ and $\widehat{\mathcal{O}_{q}}(G / H)$ with a subalgebra of $\widetilde{\mathcal{O}_{q}}(G)$ and $\widehat{\mathcal{O}_{q}}(G)$, respectively.

Lemma 4.7. $\mathcal{O}_{q}(G / H)^{\vee} \cap(q-1) \mathcal{O}_{q}(G)^{\vee}=(q-1) \mathcal{O}_{q}(G / H)^{\vee}$.

Proof. Let us choose a subset $\left\{j_{1}, \ldots, j_{n}\right\}$ in $J_{G / H}^{\text {loc }}$ as explained above for the description of $\mathcal{O}_{q}(G / H)^{\vee}$. Then, mapping $\mathcal{O}_{q}(G)^{\vee}$ and $\mathcal{O}_{q}(G / H)^{\vee}$ into their $(q-1)$-adic completions, and exploiting the descriptions of the latter via pseudobases given above, we easily get the claim.

The next result is that $\mathcal{O}_{q}(G / H)^{\vee}$ is a quantization of $U\left(\mathfrak{h}^{\perp}\right)$ :

Theorem 4.8. $\mathcal{O}_{q}(G / H)^{\vee}$ is a quantization of $U\left(\mathfrak{h}^{\perp}\right)$ as a $\mathbb{k}$-algebra, a subalgebra of $U\left(\mathfrak{g}^{*}\right)$, where $\mathfrak{h}=\operatorname{Lie}(H)$ and $\mathfrak{g}=\operatorname{Lie}(G)$.

Proof. By assumption, $H$ is coisotropic in $G$. Therefore, $\mathfrak{h}=\operatorname{Lie}(H)$ is a Lie coideal (and subalgebra) of $\mathfrak{g}=\operatorname{Lie}(G)$, and $\mathfrak{h}^{\perp}$ is a Lie subalgebra (and coideal) of $\mathfrak{g}^{*}$ (see Proposition 2.2). Thus the claim does make sense.

In order to prove the statement, we proceed much like in the proof of the fact that $\mathcal{O}_{q}(G)^{\vee} /(q-1) \mathcal{O}_{q}(G)^{\vee} \cong U\left(g^{*}\right)$, cf. [14], Theorem 4.7. The arguments being the same, we briefly recall them. 
Fix again a special subset $\left\{j_{1}, \ldots, j_{n}\right\}$ of $J_{G}$ as we did in the proof of Proposition 4.6, in particular with $j_{1}, \ldots, j_{n-h} \in J_{G / H}$. Also, set notation:

$$
\begin{aligned}
\mathcal{O}_{1}(G)^{\vee} & :=\mathcal{O}_{q}(G)^{\vee} /(q-1) \mathcal{O}_{q}(G)^{\vee}, \\
J_{G}^{\vee} & :=(q-1)^{-1} J_{G} \subseteq \mathcal{O}_{q}(G)^{\vee}, \\
j^{\vee} & :=(q-1)^{-1} j \quad \text { for all } j \in J_{G}, \\
\mathrm{t} & :=J_{G}^{\vee} \bmod (q-1) \mathcal{O}_{q}(G)^{\vee} .
\end{aligned}
$$

Taking into account that the specializations at $q=1$ of any $\mathbb{k}_{q}^{\prime}$-module and of its $(q-1)$-adic completion are the same, the above discussion gives that

$$
\left\{\prod_{s=1}^{n}\left(j_{s}^{\vee}\right)^{e_{s}} \bmod (q-1) \mathcal{O}_{q}(G)^{\vee} \mid\left(e_{1}, \ldots, e_{n}\right) \in \mathbb{N}^{n}\right\}
$$

is a $\mathbb{k}$-basis of $\mathcal{O}_{1}(G)^{\vee}$. Similarly, $\left\{j_{1}^{\vee}, \ldots, j_{n}^{\vee}\right\}$ is a $\mathbb{k}$-basis of $\mathrm{t}$.

Now, $j_{\mu} j_{v}-j_{v} j_{\mu} \in(q-1) J_{G}$ (for $\mu, v \in\{1, \ldots, n\}$ ) implies that

$$
j_{\mu} j_{v}-j_{v} j_{\mu}=(q-1) \sum_{s=1}^{n} c_{s} j_{s}+(q-1)^{2} \gamma_{1}+(q-1) \gamma_{2}
$$

for some $c_{s} \in \mathbb{k}_{q}^{\prime}, \gamma_{1} \in J_{G}$ and $\gamma_{2} \in J_{G}^{2}$. Therefore

$$
\begin{aligned}
{\left[j_{\mu}^{\vee}, j_{v}^{\vee}\right] } & :=j_{\mu}^{\vee} j_{v}^{\vee}-j_{v}^{\vee} j_{\mu}^{\vee} \\
& =\sum_{s=1}^{n} c_{s} j_{s}^{\vee}+\gamma_{1}+(q-1) \gamma_{2}^{\vee} \equiv \sum_{s=1}^{n} c_{s} j_{s}^{\vee} \bmod (q-1) \mathcal{O}_{q}(G)^{\vee}
\end{aligned}
$$

where $\gamma_{2}^{\vee}:=(q-1)^{-2} \gamma_{2} \in(q-1)^{-2}\left(J_{G}^{\vee}\right)^{2} \subseteq \mathcal{O}_{q}(G)^{\vee}$; thus $t$ is a Lie subalgebra of $\mathcal{O}_{1}(G)^{\vee}$. But then we have $\mathcal{O}_{1}(G)^{\vee} \cong U(\mathrm{t})$ as Hopf algebras by the above description of $\mathcal{O}_{1}(G)^{\vee}$ and PBW theorem.

Next, the specialization map $p^{\vee}: \mathcal{O}_{q}(G)^{\vee} \rightarrow \mathcal{O}_{1}(G)^{\vee}=U(\mathrm{t})$ restricts to $\eta: J_{G}^{\vee} \rightarrow \mathrm{t}:=J_{G}^{\vee} \bmod (q-1) \mathcal{O}_{q}(G)^{\vee}$. Moreover, multiplication by $(q-1)^{-1}$ yields a $\mathbb{k}_{q}^{\prime}$-module isomorphism $\mu: J_{G} \stackrel{\cong}{\leftrightarrows} J_{G}^{\vee}$. Consider the natural projection map $\rho: J_{1} \rightarrow J_{1} / J_{1}^{2}=\mathrm{g}^{*}$, and let $\nu: \mathrm{g}^{*} \hookrightarrow J_{1}$ be a section of $\rho$. The specialization map $p: \mathcal{O}_{q}(G) \rightarrow \mathcal{O}(G)$ restricts to $p^{\prime}: J_{G} \rightarrow J_{1}$, and we fix a section $\gamma: J_{1} \hookrightarrow J_{G}$ of $p^{\prime}$. Then the composition map $\sigma:=\eta \circ \mu \circ \gamma \circ v: \mathrm{g}^{*} \longrightarrow \mathrm{t}$ is a well-defined Lie bialgebra isomorphism, independent of the choice of $v$ and $\gamma$.

So far we did not exploit our special choice of the subset $\left\{j_{1}, \ldots, j_{n}\right\}$ : we do it now to prove that $\mathrm{t}=\mathfrak{h}^{\perp}$. In fact, the analysis above to prove that $\sigma: \mathrm{g}^{*} \cong \mathrm{t}$ shows also that the unital subalgebra

$$
\mathcal{O}_{1}(G / H)^{\vee}:=\mathcal{O}_{q}(G / H)^{\vee} \bmod (q-1) \mathcal{O}_{q}(G)^{\vee}
$$


of $U\left(\mathfrak{g}^{*}\right)$ is generated by $\eta\left(\left\{j_{1}^{\vee}, \ldots, j_{n-h}^{\vee}\right\}\right)$, and

$$
\begin{aligned}
\eta\left(\left\{j_{1}^{\vee}, \ldots, j_{n-h}^{\vee}\right\}\right) & =(\eta \circ \mu)\left(\left\{j_{1}, \ldots, j_{n-h}\right\}\right) \\
& =(\eta \circ \mu \circ \gamma)\left(\left\{y_{1}, \ldots, y_{n-h}\right\}\right) \\
& =(\eta \circ \mu \circ \gamma \circ v)\left(\left\{\bar{y}_{1}, \ldots, \bar{y}_{n-h}\right\}\right)=\sigma\left(\left\{\bar{y}_{1}, \ldots, \bar{y}_{n-h}\right\}\right)
\end{aligned}
$$

where $\bar{y}_{s}:=y_{s} \bmod \left(J_{1}^{2}\right)(s=1, \ldots, n-h)$. Therefore $\mathcal{O}_{1}(G / H)^{\vee}$ is the subalgebra of $U\left(\mathrm{~g}^{*}\right)$ generated by the $\mathbb{k}$-span of $\left\{\bar{y}_{1}, \ldots, \bar{y}_{n-h}\right\}$.

Finally, the $\mathbb{k}$-span of $\left\{\bar{y}_{1}, \ldots, \bar{y}_{n-h}\right\}$ coincides with the subspace $\mathfrak{h} \perp$ of $\mathrm{g}^{*}$. Indeed, as $\mathcal{O}(G / H)$ is the algebra of semi-invariant functions on $G$, every $y_{s}$ is a $(H$-)semi-invariant function on $G$ : but it also vanishes at $e \in H$, hence by $H$-semiinvariance it vanishes on all of $H$. When mapping $y_{s}$ to $\bar{y}_{s} \in J_{1}$, then, it is mapped into $\mathfrak{h}^{\perp}$. Thus the whole $\mathbb{k}$-span of $\left\{\bar{y}_{1}, \ldots, \bar{y}_{n-h}\right\}$ is contained in $\mathfrak{h}^{\perp}$, hence coincides with it by dimension equality.

The outcome is that $\mathcal{O}_{1}(G / H)^{\vee}$ is the subalgebra of $U\left(\mathfrak{g}^{*}\right)$ generated by $\mathfrak{h}^{\perp}$, which is a Lie subalgebra of $\mathrm{g}^{*}$, so $\mathcal{O}_{1}(G / H)^{\vee}=U\left(\mathfrak{h}^{\perp}\right)$.

We now wish to explore the nature of $\widehat{\mathcal{O}_{q}}(G / H)^{\vee}$ as a "quantum homogeneous space". We start with an important observation on the extensions of the comultiplication $\Delta$ in $\mathcal{O}_{q}(G)$ to the new algebras we have defined.

Remark 4.9. Let $\Delta: \mathcal{O}_{q}(G) \rightarrow \mathcal{O}_{q}(G) \otimes \mathcal{O}_{q}(G)$ be the comultiplication in $\mathcal{O}_{q}(G)$. Then $\Delta$ can be uniquely (and canonically) extended to a coassociative morphism of topological algebras

$$
\widetilde{\Delta}: \widetilde{\mathcal{O}_{q}}(G) \rightarrow \widetilde{\mathcal{O}_{q}}(G) \widetilde{\otimes} \widetilde{\mathcal{O}_{q}}(G),
$$

where again $\widetilde{\mathcal{O}_{q}}(G)$ is the $I_{G}$-adic completion of $\mathcal{O}_{q}(G)$ and $\widetilde{\otimes}$ is the $I_{G \otimes}$-adic completion of $\mathcal{O}_{q}(G) \otimes \mathcal{O}_{q}(G)$, with $I_{G \otimes}:=I_{G} \otimes \mathcal{O}_{q}(G)+\mathcal{O}_{q}(G) \otimes I_{G}$. Even more, such a $\widetilde{\Delta}$ actually restricts to a coassociative algebra morphism (we use the same symbol to denote it):

$$
\widetilde{\Delta}: \mathcal{O}_{q}(G)\left[d^{-1}\right] \rightarrow \mathcal{O}_{q}(G)\left[d^{-1}\right] \widetilde{\otimes} \mathcal{O}_{q}(G)\left[d^{-1}\right]
$$

In fact, as $d$ is a quantum section we have (see Definition 3.12)

$$
\Delta(d)=d \otimes d+\sum_{i} h_{i} \otimes k_{i} \quad \text { for some } h_{i} \in \mathcal{O}_{q}(G), k_{i} \in I_{q}(H) .
$$

Since $d$ is Ore, we can re-write $\widetilde{\Delta}(d)=\Delta(d)\left(d^{-1} \otimes d^{-1}\right)(d \otimes d)$, which reads

$$
\widetilde{\Delta}(d)=\left(1 \otimes 1+\sum_{i} h_{i} d^{-1} \otimes k_{i} d^{-1}\right)(d \otimes d) .
$$


This in turn implies that

$$
\begin{aligned}
\widetilde{\Delta}\left(d^{-1}\right)=\widetilde{\Delta}(d)^{-1} & =(d \otimes d)^{-1}\left(1 \otimes 1+\sum_{i} h_{i} d^{-1} \otimes k_{i} d^{-1}\right)^{-1} \\
& =\left(d^{-1} \otimes d^{-1}\right) \sum_{n=0}^{+\infty}(-1)^{n}\left(\sum_{i} h_{i} d^{-1} \otimes k_{i} d^{-1}\right)^{n},
\end{aligned}
$$

where the bottom term does belong to $\mathcal{O}_{q}(G)\left[d^{-1}\right] \widetilde{\otimes} \mathcal{O}_{q}(G)\left[d^{-1}\right]$, as expected, because $k_{i} \in I_{q}(H) \subset I_{G}$ (for every $i$ ), hence the last formal series above is convergent in the $I_{G \otimes}$-adic topology.

Let us now turn our attention to the algebra $\mathcal{O}_{q}(G)^{\vee}$ and its $(q-1)$-adic completion $\widehat{\mathcal{O}_{q}}(G)^{\vee}$. By construction (cf. [14]), the coproduct of $\mathcal{O}_{q}(G)^{\vee}$, hence of $\widehat{\mathcal{O}_{q}}(G)^{\vee}$ too, is induced by the coproduct $\Delta$ of $\mathcal{O}_{q}(G)$. Note that the coproduct $\widehat{\Delta}$ of $\widehat{\mathcal{O}_{q}}(G)^{\vee}$ takes values in the topological tensor product $\widehat{\mathcal{O}_{q}}(G)^{\vee} \widehat{\otimes} \widehat{\mathcal{O}_{q}}(G)^{\vee}$, which by definition is the $(q-1)$-adic completion of the algebraic tensor product $\widehat{\mathcal{O}_{q}}(G)^{\vee} \otimes \widehat{\mathcal{O}_{q}}(G)^{\vee}-$ and coincides, moreover, with the $(q-1)$-adic completion of $\mathcal{O}_{q}(G)^{\vee} \otimes \mathcal{O}_{q}(G)^{\vee}$.

We are ready to move another key step.

Proposition 4.10. $\widehat{\mathcal{O}_{q}}(G / H)^{\vee}$ is a left coideal of $\widehat{\mathcal{O}_{q}}(G)^{\vee}$.

Proof. We want to show that the coproduct $\widehat{\Delta}$ maps $\widehat{\mathcal{O}_{q}}(G / H)^{\vee}$ into the topological tensor product $\widehat{\mathcal{O}_{q}}(G)^{\vee} \widehat{\otimes} \widehat{\mathcal{O}_{q}}(G / H)^{\vee}$.

We first observe that

$$
\widetilde{\Delta}\left(d^{-1}\right) \in \mathcal{O}_{q}(G)\left[d^{-1}\right] \widetilde{\otimes} \mathcal{O}_{q}(G / H)\left[d^{-1}\right] .
$$

This is because $\mathcal{O}_{q}(G / H)$ is a left coideal of $\mathcal{O}_{q}(G)$, cf. Proposition 3.10, Theorem 3.15. Hence the elements $k_{i}$ occurring in formula (4.2) can be taken to belong to $\mathcal{O}_{q}(G / H)$.

Even more precisely, as the $\mathcal{O}_{q}(G)$-coaction on $\mathcal{O}_{q}(G / H)$ via $\Delta$ is graded (by Theorem $3.15(\mathrm{c})$ ), all the $k_{i}$ 's have degree 1 , like $d$ itself. Thus, the series occurring in (4.3) in fact belongs to $\mathcal{O}_{q}(G)\left[d^{-1}\right] \widetilde{\otimes} \mathcal{O}_{q}^{\text {loc }}(G / H)$. To sum up, we obtain that

$$
\widetilde{\Delta}\left(d^{-1}\right)=\left(d^{-1} \otimes d^{-1}\right) \cdot \delta_{\otimes} \quad \text { with } \delta_{\otimes} \in \mathcal{O}_{q}(G)\left[d^{-1}\right] \tilde{\otimes} \mathcal{O}_{q}^{\text {loc }}(G / H) .
$$

Since the coaction $\Delta: \mathcal{O}_{q}(G / H) \rightarrow \mathcal{O}_{q}(G) \otimes \mathcal{O}_{q}(G / H)$ is grading-preserving and product-preserving, the definitions of $\mathcal{O}_{q}^{\text {loc }}(G / H)$ and $\mathcal{O}_{q}^{\text {loc }}(G)$ and (4.4) together yield that

$$
\tilde{\Delta}\left(\mathcal{O}_{q}^{\text {loc }}(G / H)\right) \subseteq \mathcal{O}_{q}(G)\left[d^{-1}\right] \tilde{\otimes} \mathcal{O}_{q}^{\text {loc }}(G / H) .
$$

We described above, for $A \in\left\{\mathcal{O}_{q}(G / H), \mathcal{O}_{q}(G)\right\}$, the completions of the algebras $A$ and $A^{\vee}$ with respect to the $I_{G}$-adic or the $(q-1)$-adic topology. Using that, or an 
entirely similar analysis, we see also that

$$
\mathcal{O}_{q}(G)\left[d^{-1}\right] \tilde{\otimes} \mathcal{O}_{q}^{\text {loc }}(G / H) \subseteq \widehat{\mathcal{O}_{q}}(G)^{\vee} \hat{\otimes} \widehat{\mathcal{O}_{q}}(G / H)^{\vee} .
$$

In short, this is because $I_{G} \subseteq(q-1) \mathcal{O}_{q}(G)^{\vee}$ and $I_{G / H} \subseteq(q-1) \mathcal{O}_{q}(G / H)^{\vee}$. Also it is easily seen that

$$
\widetilde{\Delta}\left(I_{G / H}^{\mathrm{loc}}\right) \subseteq \mathcal{O}_{q}(G)\left[d^{-1}\right] \widetilde{\otimes} I_{G / H}^{\mathrm{loc}}+I_{G} \tilde{\otimes} \mathcal{O}_{q}^{\mathrm{loc}}(G / H) .
$$

This along with (4.5) immediately implies that $\widehat{\Delta}\left((q-1)^{-1} I_{G / H}^{\mathrm{loc}}\right) \subseteq \mathcal{O}_{q}(G)\left[d^{-1}\right] \widehat{\otimes}(q-1)^{-1} I_{G / H}^{\mathrm{loc}}+(q-1)^{-1} I_{G} \hat{\otimes} \mathcal{O}_{q}^{\mathrm{loc}}(G / H)$, which in turn yields, by the very definition of $\mathcal{O}_{q}(G)^{\vee}$ and $\mathcal{O}_{q}(G / H)^{\vee}$, that

$$
\widehat{\Delta}\left(\mathcal{O}_{q}(G / H)^{\vee}\right) \subseteq \mathcal{O}_{q}(G)^{\vee} \hat{\otimes} \mathcal{O}_{q}(G / H)^{\vee} .
$$

Finally, taking $(q-1)$-adic completions on both sides, and also noting that $\mathcal{O}_{q}(G)^{\vee} \hat{\otimes} \mathcal{O}_{q}(G / H)^{\vee}=\widehat{\mathcal{O}_{q}}(G)^{\vee} \hat{\otimes} \widehat{\mathcal{O}_{q}}(G / H)^{\vee}$, we get

$$
\widehat{\Delta}\left(\widehat{\mathcal{O}_{q}}(G / H)^{\vee}\right) \subseteq \mathcal{O}_{q}(G)^{\vee} \widehat{\otimes} \widehat{\mathcal{O}_{q}}(G / H)^{\vee} .
$$

In the end, we obtain the main result of this section:

Theorem 4.11. $\widehat{\mathcal{O}_{q}}(G / H)^{\vee}$ is a quantization of $U\left(\mathfrak{h}^{\perp}\right)$ as a subalgebra and left coideal of $U\left(\mathrm{~g}^{*}\right)$. In other words, $\widehat{\mathcal{O}_{q}}(G / H)^{\vee}$ is an infinitesimal quantization of the coisotropic subgroup $H^{\perp}$ of $G^{*}$.

Proof. Just collect the previous results. First we have

$$
\widehat{\mathcal{O}_{q}}(G / H)^{\vee} \cap(q-1) \widehat{\mathcal{O}_{q}}(G)^{\vee}=(q-1) \widehat{\mathcal{O}_{q}}(G / H)^{\vee}
$$

as an easy consequence of Lemma 4.7. Then, by Theorem 4.8 and by the fact that $\left.\widehat{\mathcal{O}_{q}}(G / H)^{\vee}\right|_{q=1}=\left.\mathcal{O}_{q}(G / H)^{\vee}\right|_{q=1}$, we have that the specialization of $\widehat{\mathcal{O}_{q}}(G / H)^{\vee}$ is $U\left(\mathfrak{h}^{\perp}\right)$. Moreover, Proposition 4.10 shows that the subalgebra $\widehat{\mathcal{O}_{q}}(G / H)^{\vee}$ of $\widehat{\mathcal{O}_{q}}(G)^{\vee}$ is also a left coideal. Therefore, $\widehat{\mathcal{O}_{q}}(G / H)^{\vee}$ is an infinitesimal quantization of $H^{\perp}$ in the standard sense.

Remark 4.12. The construction of $\widehat{\mathcal{O}_{q}}(G / H)^{\vee}$ is functorial in the following sense. For a fixed $\mathcal{O}_{q}(G)$ every $\mathcal{O}_{q}(G / H)$ is uniquely characterized by the pair $\left(\pi_{H}, d_{H}\right)$ given by the projection $\pi_{H}: \mathcal{O}_{q}(G) \rightarrow \mathcal{O}_{q}(H)$ and the quantum section $d_{H} \in$ $\mathcal{O}_{q}(G)$. The natural notion of morphism among such pairs, say $\left(\pi_{H^{\prime}}, d_{H^{\prime}}\right) \rightarrow$ $\left(\pi_{H^{\prime \prime}}, d_{H^{\prime \prime}}\right)$, can be cast into the form a Hopf algebra endomorphism $\phi$ of $\mathcal{O}_{q}(G)$ such that $\phi\left(\operatorname{Ker}\left(\pi_{H^{\prime}}\right)\right) \subseteq \operatorname{Ker}\left(\pi_{H^{\prime \prime}}\right)-$ or $\phi\left(I_{q}\left(H^{\prime}\right)\right) \subseteq I_{q}\left(H^{\prime \prime}\right)-$ and $\phi\left(d_{H^{\prime}}\right)=d_{H^{\prime \prime}}$. 
Then, one defines ()$^{\vee}$ on morphisms by scalar extension followed by restriction; proving the functoriality is a matter of bookkeeping. More in general, one might decide not to fix $\mathcal{O}_{q}(G)$, nor even $G$. Then morphisms $\phi: \mathcal{O}_{q}\left(G^{\prime}\right) \rightarrow \mathcal{O}_{q}\left(G^{\prime \prime}\right)$ take the place of the endomorphisms of (the single) $\mathcal{O}_{q}(G)$ in the recipe above, yet ()$^{\vee}$ is defined again on morphisms via scalar extension and restriction, and one has to exploit the functoriality of ()$^{\vee}$ over quantum groups $\mathcal{O}_{q}(G)$.

\section{Examples: Quantum Grassmannians and quantum flag varieties}

In this section we want to examine in detail some examples of quantum homogeneous spaces and apply the quantum duality principle recipe to them. We start with the quantum Grassmannian.

5.1. The quantum Grassmannian as quantum projective homogenous space. Let us recall the classical setting.

Let $G=\mathrm{GL}_{n}(\mathbb{k})$ and let $H=P$ a (standard) maximal parabolic subgroup, say

$$
P=\left\{\left(\begin{array}{cc}
A & B \\
0 & D
\end{array}\right) \mid A \in \mathrm{GL}_{r}(\mathbb{k}), B \in \mathrm{Mat}_{r, n-r}(\mathbb{k}), D \in \mathrm{GL}_{n-r}(\mathbb{k})\right\} .
$$

First we want to give a very ample line bundle on the homogeneous space $G / P$ the Grassmann variety - that realizes the classical Plücker embedding into a projective space, following the recipe in Section 2.2.

Let $I=\left(i_{1}, \ldots, i_{r}\right), 1 \leq i_{1}<\cdots<i_{r} \leq n$. Define

$$
d^{I}: g=\left(x_{i j}\right) \mapsto d^{I}(g):=\sum_{\sigma \in \mathcal{S}_{r}}(-1)^{\ell(\sigma)} x_{i_{1}, \sigma(1)} \ldots x_{i_{r}, \sigma(r)}
$$

as the function corresponding to the determinant of the minor of a matrix $g=$ $\left(x_{i j}\right) \in \mathrm{GL}_{n}(\mathrm{k})$ obtained by taking rows $i_{1}, \ldots, i_{r}$ and columns $1, \ldots, r$. Then $d^{I} \in \mathcal{O}\left(\mathrm{GL}_{n}(\mathbb{k})\right)$ for all $I$, i.e., these are regular functions on $\mathrm{GL}_{n}(\mathbb{k})$.

If $I_{0}:=(1, \ldots, r)$, then $d^{I_{0}}$ restricts to a map (with same name)

$$
d^{I_{0}}: P \rightarrow \mathbb{k}^{\times}, \quad M:=\left(\begin{array}{cc}
A & B \\
0 & D
\end{array}\right) \mapsto d^{I_{0}}(M)=\operatorname{det}(A),
$$

which is a character of $P$. One checks that the line bundle $\mathscr{L}$ associated to $d^{I_{0}}$ is very ample, and it provides an embedding of $G / P$ into a projective space, following the recipe in Section 2.2. Algebraically, this means that the graded algebra $\mathcal{O}(G / P)$ is realized as embedded into $\mathcal{O}(G)$ as

$$
\mathcal{O}(G / P)=\bigoplus_{n \geq 0} \mathcal{O}(G / P)_{n}=\bigoplus_{n \geq 0} H^{0}\left(G / P, \mathscr{L}^{\otimes n}\right)
$$

In particular, one can easily verify, for any set $I$ of $r$ rows, that

$$
d^{I}(g p)=d^{I_{0}}(p) d^{I}(g) \quad \text { for all } g \in \mathrm{GL}_{n}(\mathbb{k}), p \in P,
$$


i.e., $d^{I}$ is $d^{I_{0}}$-semi-invariant of degree 1 . In addition, one proves that the $d^{I}$ 's form a $\mathbb{k}$-basis of the space $\mathcal{O}(G / P)_{1}$ of semi-invariants of degree 1 (cf. [23]).

On the other hand, the spaces $\mathcal{O}(G / P)_{n}=H^{0}\left(G / P, \mathscr{L}^{\otimes n}\right)$ are in one-to-one correspondence - up to twisting by any integral power of det (i.e., by any character of $\left.\mathrm{GL}_{n}(\mathbb{k})\right)$ - with the irreducible representations of $\mathrm{GL}_{n}(\mathbb{k})$.

We will now see that this picture extends to the quantum setup.

Let $\mathcal{O}_{q}\left(M_{n}\right)$ be the unital associative algebra over $\mathbb{k}_{q}=\mathbb{k}\left[q, q^{-1}\right]$ with generators $x_{i j}($ for $1 \leq i, j \leq n)$ and relations

$$
\begin{array}{ll}
x_{i j} x_{i k}=q x_{i k} x_{i j}, x_{j i} x_{k i}=q x_{k i} x_{j i} & \text { for all } j<k \text { and all } i, \\
x_{i j} x_{k l}=x_{k l} x_{i j} & \text { for all } i<k, j>l \text { or } i>k, j<l, \\
x_{i j} x_{k l}-x_{k l} x_{i j}=\left(q-q^{-1}\right) x_{k j} x_{i l} & \text { for all } i<k, j<l
\end{array}
$$

(the so-called "Manin relations"). This algebra bears also a structure of $\mathbb{k}_{q}$-bialgebra, whose coproduct and counit are given by

$$
\Delta\left(x_{i j}\right)=\sum_{k=1}^{n} x_{i k} \otimes x_{k j}, \quad \epsilon\left(x_{i j}\right)=\delta_{i j} \quad \text { for all } i, j .
$$

Define the "quantum determinant" (of order $n$ ) $\operatorname{det}_{q}$ as

$$
\operatorname{det}_{q}:=\sum_{\sigma \in S_{n}}(-q)^{\ell(\sigma)} x_{1, \sigma(1)} \ldots x_{n, \sigma(n)} \in \mathcal{O}_{q}\left(M_{n}\right) .
$$

One proves that $\operatorname{det}_{q}$ belongs to the center of $\mathcal{O}\left(M_{n}\right)$, and it is group-like, i.e., $\Delta\left(\operatorname{det}_{q}\right)=\operatorname{det}_{q} \otimes \operatorname{det}_{q}$ and $\epsilon\left(\operatorname{det}_{q}\right)=1$.

More generally, for any $1 \leq r \leq n$ and for any choice of $r$-tuples of increasing indices $I=\left(i_{1}, \ldots, i_{r}\right)$ and $J=\left(j_{1}, \ldots, j_{r}\right)$, we define the "quantum determinant of the minor $(I, J)$ ", i.e., of the minor (of the matrix with entries the $x_{i j}$ 's) whose sets of rows and columns are $I$ and $J$, namely

$$
D_{J}^{I}:=\sum_{\sigma \in \mathcal{S}_{r}}(-q)^{\ell(\sigma)} x_{i_{1}, j_{\sigma(1)}} \ldots x_{i_{r}, i_{\sigma(r)}}
$$

These satisfy (cf. [19], §9.2.2) the following quantum analogue of well-known classical identities (e.g., the first one is analogous to Binet's theorem):

$$
\Delta\left(D_{J}^{I}\right)=\sum_{K} D_{K}^{I} \otimes D_{J}^{K}, \quad \epsilon\left(D_{J}^{I}\right)=\delta_{I, J}
$$

Since $\operatorname{det}_{q}$ is central in $\mathcal{O}_{q}\left(M_{n}\right)$, it is a Ore element as well, and we can consider the enlarged algebra $\mathcal{O}_{q}\left(\mathrm{GL}_{n}\right):=\mathcal{O}_{q}\left(M_{n}\right)\left[\operatorname{det}_{q}^{-1}\right]$ obtained from $\mathcal{O}_{q}\left(M_{n}\right)$ by formally inverting $\operatorname{det}_{q}$. Then - see [19] again - the bialgebra structure of $\mathcal{O}_{q}\left(M_{n}\right)$ uniquely extends to $\mathcal{O}_{q}\left(\mathrm{GL}_{n}\right)$; even more, the latter then is a Hopf algebra. In fact, 
by construction $\mathcal{O}_{q}\left(\mathrm{GL}_{n}\right)$ is a quantum group, namely a quantization of $\mathrm{GL}_{n}(\mathbb{k})$ in the sense of Definition 3.1.

We shall again denote by $x_{i j}$ the images in $\mathcal{O}_{q}\left(\mathrm{GL}_{n}\right)$ of the generators $x_{i j}$ of $\mathcal{O}_{q}\left(M_{n}\right)$. Similarly, we shall denote by $D_{J}^{I}$ the images in $\mathcal{O}_{q}\left(\mathrm{GL}_{n}\right)$ of the "quantum minors" of $\mathcal{O}_{q}\left(M_{n}\right)$ : then they again enjoy (5.2) and (5.3). Letting $J_{0}:=$ $(1, \ldots, r)=: I_{0}$, hereafter we shall set $D^{I}:=D_{J_{0}}^{I}$.

The specialization (at $q=1$ ) of any quantum minor $D_{J}^{I}$ is the corresponding classical minor $d_{J}^{I}$ (on the same sets of rows and columns); in particular, every $D^{I}$ specializes to $d^{I}-$ see (5.1) - and, among them, $D^{I_{0}}$ to $d^{I_{0}}$.

We define the quantum parabolic subgroup $\mathcal{O}_{q}(P)$ as the quotient algebra

$$
\mathcal{O}_{q}(P):=\mathcal{O}_{q}\left(\mathrm{GL}_{n}\right) /\left(\left\{x_{i j} \mid r+1 \leq i \leq n ; 1 \leq j \leq r\right\}\right) .
$$

One can easily check that this $\mathcal{O}_{q}(P)$ is in fact a Hopf algebra quotient. Thus the natural projection map $\pi: \mathcal{O}_{q}(G) \rightarrow \mathcal{O}_{q}(P)$ is a Hopf algebra epimorphism. Therefore, $\mathcal{O}_{q}(P)$ is a quantum Poisson subgroup of $\mathcal{O}_{q}(G)=\mathcal{O}_{q}\left(\mathrm{GL}_{n}(\mathbb{k})\right)$ in the sense of Definition 3.3, whose specialization is $\mathcal{O}(P)$.

We are now in a position to appreciate the first important fact, in the present setting, about quantum minors:

Lemma 5.1. The quantum minor $D^{I_{0}}$ is a quantum section of the line bundle on $G / P$ given by $d^{I_{0}}$ in the sense of Definition 3.12 .

Proof. Using the first identity in (5.3) one gets

$$
\Delta_{\pi}\left(D^{I_{0}}\right)=((\mathrm{id} \otimes \pi) \circ \Delta)\left(D^{I_{0}}\right)=(\mathrm{id} \otimes \pi)\left(\sum_{K} D_{K}^{I_{0}} \otimes D_{J_{0}}^{K}\right)=\sum_{K} D_{K}^{I_{0}} \otimes \overline{D_{J_{0}}^{K}}
$$

and then from this

$$
\Delta_{\pi}\left(D^{I_{0}}\right)=D_{I_{0}}^{I_{0}} \otimes \overline{D_{J_{0}}^{I_{0}}}=D^{I_{0}} \otimes \overline{D^{I_{0}}}
$$

because $\overline{D_{J_{0}}^{K}}:=\pi\left(D_{J_{0}}^{K}\right)=\delta_{K, I_{0}} \overline{D_{J_{0}}^{I_{0}}}$ by definition of $\pi$, and $D_{I_{0}}^{I_{0}}=D_{J_{0}}^{I_{0}}=D^{I_{0}}$. Therefore (see Definition 3.5) $D^{I_{0}}$ is a pre-quantum section; but $\mathcal{O}_{q}(P)$ is a quantum subgroup, so (see Proposition 3.13) $D^{I_{0}}$ is a quantum section.

Using $D^{I_{0}}$, we can perform the construction of the algebra $\mathcal{O}_{q}(G / P)$ of $D^{I_{0}}$-semiinvariants (or simply semi-invariants) as in Section 3. First we have the following result.

Lemma 5.2. The quantum minors $D^{I}$ are all semi-invariants of degree 1 , that is to say, $D^{I} \in \mathcal{O}_{q}(G / P)_{1}$ for every set of rows $I=\left(i_{1}, \ldots, i_{r}\right)$. 
Proof. Arguing as in the proof of Lemma 5.1, we prove the claim by

$$
\begin{aligned}
\Delta_{\pi}\left(D^{I}\right) & =((\mathrm{id} \otimes \pi) \circ \Delta)\left(D^{I}\right) \\
& =(\mathrm{id} \otimes \pi)\left(\sum_{K} D_{K}^{I} \otimes D_{J_{0}}^{K}\right) \\
& =\sum_{K} D_{K}^{I} \otimes \overline{D_{J_{0}}^{K}}=D_{I_{0}}^{I} \otimes \overline{D_{J_{0}}^{I_{0}}}=D^{I} \otimes \overline{D^{I_{0}}} .
\end{aligned}
$$

Roughly speaking, the outcome of this last result is that the line bundle on $G / P$ given by $d^{I_{0}}$ has enough "quantum sections" to provide a "quantum projective embedding". To be precise, the following holds:

Corollary 5.3. The space $\mathcal{O}_{q}(G / P)$ of all $D^{I_{0}}$-semi-invariants is a quantization of $\mathcal{O}(G / P)$, in the sense of Definition 3.18.

Proof. By construction every quantum minor $D^{I}$ specializes to the corresponding classical minor $d^{I}$. By Section 5.1, the latter form a basis of $\mathcal{O}(G / P)_{1}$.

This along with Lemma 5.2 proves that the natural embedding

$$
\mathcal{O}_{q}(G / P)_{1} /(q-1) \mathcal{O}_{q}(G / P)_{1} \hookrightarrow \mathcal{O}(G / P)_{1}
$$

is onto. But then, as noticed in Remark 3.19, the claim follows.

Actually, we can prove the following, much more precise result:

Proposition 5.4. The algebra $\mathcal{O}_{q}(G / P)$ is generated by the $D^{I}$ 's.

Proof. By Lemma 5.2, the $D^{I}$ 's belong to $\mathcal{O}_{q}(G / P)$. Therefore, we are only left to prove that, conversely, every semi-invariant is contained in the $\mathbb{k}$-subalgebra of $\mathcal{O}_{q}(G)$ generated by the $D^{I}$ 's.

To this end, Theorems 1.2 and 1.3 in [16] give us immediately the result if we take $\mathbb{k}(q)$ as ground ring instead of $\mathbb{k}_{q}:=\mathbb{k}\left[q, q^{-1}\right]$. Then Lemmas 3.9, 3.10, 3.11 in [12] give us our result. We now present this in detail.

We start by rewriting the Proposition 1.1 in [16] in our notation:

Let $A \stackrel{\phi}{\rightarrow} B \stackrel{\psi}{\rightarrow}$ C be a complex of $\mathbb{k}_{q}$-modules such that $C$ is torsion free. Suppose there are $\mathbb{k}_{q}$-module decompositions $A=\bigoplus_{j} A_{j}, B=\bigoplus_{j} B_{j}, C=\bigoplus_{j} C_{j}$ such that $B_{j}$ is finitely generated, and the maps $\phi$ and $\psi$ respect the decomposition, that is, $\phi\left(A_{j}\right) \subseteq B_{j}$ and $\psi\left(B_{j}\right) \subseteq C_{j}$. If the sequence $\bar{A} \rightarrow \bar{B} \rightarrow \bar{C}$ obtained by reduction modulo $(q-1)$ is exact, then so is

$$
\mathbb{k}(q) \otimes_{\mathbb{k}_{q}} A \stackrel{\phi}{\rightarrow} \mathbb{k}(q) \otimes_{\mathbb{k}_{q}} B \stackrel{\psi}{\rightarrow} \mathbb{k}(q) \otimes_{\mathbb{k}_{q}} C
$$

Let us apply this result to our special situation. 
The subalgebra $A:=\mathbb{k}_{q}\left[D^{I}\right]$ generated in $\mathcal{O}_{q}\left(\mathrm{SL}_{n}\right)$ by quantum determinants is a $\mathbb{k}_{q}$-graded module (by the degree). This fact is non-trivial and depends on the explicit form of this algebra in terms of generators and relations; see [9]. We have that an element $a \in \mathcal{O}_{q}\left(\mathrm{SL}_{n}\right)$ is in $A_{j}$ iff

$$
\sum_{(a)} a_{(1)} \otimes \bar{a}_{(2)}=a \otimes \bar{d}^{j}
$$

where $\Delta(a)=\sum_{(a)} a_{(1)} \otimes a_{(2)}$ and $\bar{x}$ denotes reduction of $x$ modulo $I_{q}(P)$ (see notation in Section 3.3).

So we can set up maps

$$
A \stackrel{\phi}{\rightarrow} \mathcal{O}_{q}\left(\mathrm{SL}_{n}\right) \stackrel{\psi}{\rightarrow} \mathcal{O}_{q}\left(\mathrm{SL}_{n}\right) \otimes \mathcal{O}_{q}(P)
$$

where $\phi$ is the inclusion and $\psi$ is defined by

$$
\psi(a)=\sum_{(a)} a_{(1)} \otimes \bar{a}_{(2)}-a \otimes \bar{d}^{j} \quad \text { for all } a \in A_{j} .
$$

One can check that all the hypotheses of the previous result, for $B:=\mathcal{O}_{q}\left(\mathrm{SL}_{n}\right)$ and $C:=\mathcal{O}_{q}\left(\mathrm{SL}_{n}\right) \otimes \mathcal{O}_{q}(P)$, are satisfied. Thus we get $\mathbb{k}(q) \otimes_{\mathbb{k}} A \cong \operatorname{Ker}(\mathrm{id} \otimes \psi)$. In other words, the semi-invariants coincide with the subalgebra generated by the quantum determinants over the $\operatorname{ring} \mathrm{k}(q)$.

We now obtain the result over $\mathbb{k}_{q}$ by Lemma 3.11 in [10], namely:

If $w X \in \mathbb{k}_{q}\left[D^{I}\right], w \in \mathbb{k}_{q}, w \neq 0$, then $X \in \mathbb{k}_{q}\left[D^{I}\right]$.

Remark 5.5. Thus, using our own recipe, we have constructed the quantum homogeneous space $\mathcal{O}_{q}(G / P)$. It is immediate to see that this is the same as the deformation of the algebra of the classical Grassmannian, along with its classical Plücker embedding, as it is described in [9] or in [28].

Finally, for the $\mathcal{O}_{q}(G)$-comodule structure of the space of semi-invariants of degree 1 , we have also the following analogue of a classical result:

Proposition 5.6. $\mathcal{O}_{q}(G / P)_{1} \cong \bigwedge_{q}\left(\mathbb{k}_{q}^{n}\right)$ as left $\mathcal{O}_{q}(G)$-comodules.

Proof. This is a direct calculation, which we sketch. By all the previous analysis, we already know that $\mathcal{O}_{q}(G / P)_{1}$ the set of all the $D^{I}$ 's forms a basis, and the left $\mathcal{O}_{q}(G)$-coaction on $\mathcal{O}_{q}(G / P)_{1}$ is given by

$$
D^{I} \mapsto \sum_{K} D_{K}^{I} \otimes D^{K} .
$$


Now consider the coaction of $\mathcal{O}_{q}(G)$ on $\bigwedge_{q}\left(\mathbb{k}_{q}^{n}\right)$ given by

$$
\begin{aligned}
& \xi_{i_{1}} \ldots \xi_{i_{r}} \mapsto \sum_{k_{1}, \ldots, k_{r}} g_{i_{1} k_{1}} \ldots g_{i_{r} k_{r}} \otimes \xi_{k_{1}} \ldots \xi_{k_{r}} \\
& \quad=\sum_{\sigma}(-q)^{\ell(\sigma)} g_{i_{1} k_{1}} \ldots g_{i_{r} k_{r}} \otimes \xi_{k_{1}^{o}} \ldots \xi_{k_{r}^{o}}=\sum_{K} D_{K}^{I} \otimes \xi_{k_{1}^{o}} \ldots \xi_{k_{r}^{o}}
\end{aligned}
$$

where $\sigma$ is the permutation reordering $k_{1}, \ldots, k_{r}$ and $K=\left(k_{1}^{o}, \ldots, k_{r}^{o}\right)$ are the same indices, but reordered. Now the result follows.

Remark 5.7. Similar arguments can be used to prove that any quantum flag variety is a "quantum projective homogeneous space" in the sense of Definition 3.18 (for details about quantum flag varieties, we refer to [11]).

For the flag of type $\left(m_{1}, \ldots, m_{s}\right)$, the quantum section $d$ to start with is

$$
d:=D^{\left(m_{1}\right)} \ldots D^{\left(m_{n}\right)}
$$

where the $D^{\left(m_{j}\right)}$ 's are the principal quantum minors of size $m_{j}$.

The proofs of all results go over exactly as in the Grassmannian case.

We now turn to the construction of the quantum big cell ring that will be crucial for the explicit construction of the QDP functor.

Definition 5.8. Let $I_{0}=(1 \ldots r), D_{0}:=D^{I_{0}}$. Put

$$
\mathcal{O}_{q}(G)\left[D_{0}^{-1}\right]:=\mathcal{O}_{q}(G)[T] /\left(T D_{0}-1, D_{0} T-1\right)
$$

We define the big cell ring $\mathcal{O}_{q}^{\text {loc }}(G / P)$ to be the $\mathbb{k}_{q}$-subalgebra of $\mathcal{O}_{q}(G)\left[D_{0}^{-1}\right]$ generated by the elements

$$
t_{i j}:=(-q)^{r-j} \Delta_{i j} D_{0}^{-1} \text { for all } i, j \text { with } 1 \leq j \leq r<i \leq n,
$$

where $\Delta_{i j}:=D^{1 \ldots \hat{j} \ldots r i}$ for all $i, j$ as above (see [10] for more details).

As in the commutative setting, we have the following result:

Proposition 5.9. $\mathcal{O}_{q}^{\mathrm{loc}}(G / P) \cong \mathcal{O}_{q}(G / P)\left[D_{0}^{-1}\right]_{\mathrm{proj}}$, where the right-hand side is the degree-zero component of $\mathcal{O}_{q}(G / P)[T] /\left(T D_{0}-1, D_{0} T-1\right)$.

Proof. In the classical setting, the analogous result is proved by the following argument: one uses the so-called "straightening relations" to get rid of the extra minors (see, for example, [8], §2). Here the argument works essentially in the same way, using the quantum straightening (or Plücker) relations (see [9], §4, [28], formula (3.2) (c) and Note I, Note II). 
Remark 5.10. As before, we have that

$$
\mathcal{O}_{q}^{\mathrm{loc}}(G / P) \cap(q-1) \mathcal{O}_{q}^{\mathrm{loc}}(G)=(q-1) \mathcal{O}_{q}^{\mathrm{loc}}(G / P) .
$$

This can be easily deduced from Remark 3.9, taking into account Proposition 5.9. As a consequence, the map

$$
\mathcal{O}_{q}^{\mathrm{loc}}(G / P) /(q-1) \mathcal{O}_{q}^{\mathrm{loc}}(G / P) \rightarrow \mathcal{O}_{q}^{\mathrm{loc}}(G) /(q-1) \mathcal{O}_{q}^{\mathrm{loc}}(G)
$$

is injective, so that the specialization map

$$
\pi_{G / P}^{\text {loc }}: \mathcal{O}_{q}^{\text {loc }}(G / P) \rightarrow \mathcal{O}_{q}^{\text {loc }}(G / P) /(q-1) \mathcal{O}_{q}^{\text {loc }}(G / P)
$$

coincides with the restriction of the specialization map

$$
\pi_{G}^{\mathrm{loc}}: \mathcal{O}_{q}^{\mathrm{loc}}(G) \rightarrow \mathcal{O}_{q}^{\mathrm{loc}}(G) /(q-1) \mathcal{O}_{q}^{\mathrm{loc}}(G) .
$$

The following proposition gives a description of the algebra $\mathcal{O}_{q}^{\text {loc }}(G / P)$ :

Proposition 5.11. The big cell ring is isomorphic to a matrix algebra

$$
\mathcal{O}_{q}^{\mathrm{loc}}(G / P) \rightarrow \mathcal{O}_{q}\left(M_{(n-r) \times r}\right), \quad t_{i j} \mapsto x_{i j},
$$

for all $1 \leq j \leq r<i \leq n$, i.e., the generators $t_{i j}$ satisfy the Manin relations.

Proof. See [10], Proposition 1.9.

Remark 5.12. The Grassmannian $\mathrm{GL}_{n} / P$ can also be realized as a similar quotient of $\mathrm{SL}_{n}$ by a suitable parabolic $P^{\prime}$ (corresponding to $P$, say). Then one can also perform all related quantum constructions - the previous and the later ones - using $\mathrm{SL}_{n}$ instead of $\mathrm{GL}_{n}$, and modifying each step as needed. To begin with, one considers

$$
\mathcal{O}_{q}\left(\mathrm{SL}_{n}\right):=\mathcal{O}_{q}\left(\mathrm{GL}_{n}\right) /\left(\operatorname{det}_{q}-1\right) \cong \mathcal{O}_{q}\left(M_{n}\right) /\left(\operatorname{det}_{q}-1\right)
$$

where $\left(\operatorname{det}_{q}-1\right)$ is the (two-sided) ideal generated by $\operatorname{det}_{q}-1$, which is again a Hopf algebra, for the quotient structure from either $\mathcal{O}_{q}\left(\mathrm{GL}_{n}\right)$ or $\mathcal{O}_{q}\left(M_{n}\right)$. This is a quantization of $\mathrm{SL}_{n}(\mathbb{k})$ in the sense of Definition 3.1, for which we can consider again quantum minors and a corresponding $\mathcal{O}_{q}(P)$ as before. Then all this can be used to give an alternative definition of $\mathcal{O}_{q}(G / P)=\mathcal{O}_{q}\left(\mathrm{SL}_{n} / P^{\prime}\right)$ and of all what was considered above. Similarly, all constructions and results of Section 5.2 hereafter can be carried on using $\mathcal{O}_{q}\left(\mathrm{SL}_{n}\right)$ - and its related gadgets - instead of $\mathcal{O}_{q}\left(\mathrm{GL}_{n}\right)$.

Finally, similar considerations hold as well for the quantum flag varieties mentioned in Remark 5.7. 
5.2. QDP for quantum Grassmannians. We now turn to the quantum duality principle applied explicitly to the quantum homogeneous spaces constructed above. We start with Grassmannians.

Let us first explicitly describe the Poisson structure of the algebraic group $\mathrm{GL}_{n}$. Starting from $\mathcal{O}_{q}\left(\mathrm{GL}_{n}\right)$, as usual the classical algebra $\mathcal{O}\left(\mathrm{GL}_{n}\right)$ inherits from the former a Poisson bracket, which makes it into a Poisson Hopf algebra, so that $\mathrm{GL}_{n}$ becomes a Poisson group (see Remark 3.2 (2)). We want to describe now this Poisson bracket. Recall that

$$
\mathcal{O}\left(\mathrm{GL}_{n}\right)=\mathbb{k}\left[\left\{\bar{x}_{i j}\right\}_{i, j=1, \ldots, n}\right]\left[\operatorname{det}^{-1}\right]=\mathbb{k}\left[\left\{\bar{x}_{i j}\right\}_{i, j=1, \ldots, n}\right][t] /(t \operatorname{det}-1)
$$

where det $:=\operatorname{det}\left(\bar{x}_{i, j}\right)_{i, j=1, \ldots, n}$ is the usual determinant. Setting $\bar{x}=p(x)$ for $p: \mathcal{O}_{q}\left(\mathrm{GL}_{n}\right) \rightarrow \mathcal{O}\left(\mathrm{GL}_{n}\right)$, the Poisson structure is given (as usual) by

$$
\{\bar{a}, \bar{b}\}:=\left.(q-1)^{-1}(a b-b a)\right|_{q=1} \quad \text { for all } \bar{a}, \bar{b} \in \mathcal{O}\left(\mathrm{GL}_{n}\right) .
$$

In terms of generators, we have

$$
\begin{gathered}
\left\{\bar{x}_{i j}, \bar{x}_{i k}\right\}=\bar{x}_{i j} \bar{x}_{i k} \quad \text { for all } j<k, \quad\left\{\bar{x}_{i j}, \bar{x}_{\ell k}\right\}=0 \quad \text { for all } i<\ell, k<j \\
\left\{\bar{x}_{i j}, \bar{x}_{\ell j}\right\}=\bar{x}_{i j} \bar{x}_{\ell j} \quad \text { for all } i<\ell, \quad\left\{\bar{x}_{i j}, \bar{x}_{\ell k}\right\}=2 \bar{x}_{i j} \bar{x}_{\ell k} \quad \text { for all } i<\ell, j<k, \\
\left\{\operatorname{det}^{-1}, \bar{x}_{i j}\right\}=0, \quad\left\{\operatorname{det}, \bar{x}_{i j}\right\}=0 \quad \text { for all } i, j=1, \ldots, n .
\end{gathered}
$$

As $\mathrm{GL}_{n}$ is a Poisson Lie group, its Lie algebra $\mathrm{gl}_{n}$ has a Lie bialgebra structure (see [3], p. 24). To describe it, let us denote with $\mathrm{m}_{i j}$ the elementary matrices, which form a basis of $\mathrm{gl}_{n}$. Define (for all $i=1, \ldots, n-1, j=1, \ldots, n$ )

$$
e_{i}:=\mathrm{m}_{i, i+1}, \quad g_{j}:=\mathrm{m}_{j, j}, \quad f_{i}:=\mathrm{m}_{i+1, i}, \quad h_{i}:=g_{i}-g_{i+1} .
$$

Then $\left\{e_{i}, f_{i}, g_{j} \mid i=1, \ldots, n-1, j=1, \ldots, n\right\}$ is a set of Lie algebra generators of $\mathfrak{g l}_{n}$, and a Lie cobracket is defined on $\mathrm{gl}_{n}$ by

$\delta\left(e_{i}\right)=h_{i} \otimes e_{i}-e_{i} \otimes h_{i}, \quad \delta\left(g_{j}\right)=0, \quad \delta\left(f_{i}\right)=h_{i} \otimes f_{i}-f_{i} \otimes h_{i} \quad$ for all $i, j$.

This cobracket makes $\mathrm{gl}_{n}$ itself into a Lie bialgebra: this is the so-called standard Lie bialgebra structure on $\mathfrak{g l}_{n}$. It follows immediately that $U\left(\mathfrak{g l}_{n}\right)$ is a co-Poisson Hopf algebra, whose co-Poisson bracket is the (unique) extension of the Lie cobracket of $\mathrm{gl}_{n}$ while the Hopf structure is the standard one.

Similar constructions hold for the group $\mathrm{SL}_{n}$. One simply drops the generator $d^{-1}$, imposes the relation $d=1$ in the description of $\mathcal{O}\left(\mathrm{SL}_{n}\right)$, and replaces the $g_{s}$ 's with the $h_{i}$ 's $(i=1, \ldots, n)$ when describing $\mathfrak{s l}_{n}$.

Since $\mathrm{gl}_{n}$ is a Lie bialgebra, its dual space $\mathrm{gl}_{n}^{*}$ admits a Lie bialgebra structure, which is dual to that of $\mathrm{gl}_{n}$. Let $\left\{\mathrm{e}_{i j}:=\mathrm{m}_{i j}^{*} \mid i, j=1, \ldots, n\right\}$ be the basis of $\mathrm{gl}_{n}^{*}$ 
dual to the basis of elementary matrices for $\mathfrak{g l}_{n}$. As a Lie algebra, $\mathfrak{g} \mathfrak{l}_{n}^{*}$ can be realized as the subset of $\mathfrak{g l}_{n} \oplus \mathfrak{g l}_{n}$ of all pairs

$$
\left(\left(\begin{array}{cccc}
-m_{11} & 0 & \ldots & 0 \\
m_{21} & -m_{22} & \ldots & 0 \\
\vdots & \vdots & \vdots & \vdots \\
m_{n-1,1} & m_{n-1,2} & \ldots & 0 \\
m_{n, 1} & m_{n, 2} & \ldots & -m_{n, n}
\end{array}\right),\left(\begin{array}{ccccc}
m_{11} & m_{12} & \ldots & m_{1, n-1} & m_{1, n} \\
0 & m_{22} & \ldots & m_{2, n-1} & m_{2, n} \\
\vdots & \vdots & \vdots & \vdots & \vdots \\
0 & 0 & \ldots & m_{n-1, n-1} & m_{n-1, n} \\
0 & 0 & \ldots & 0 & m_{n, n}
\end{array}\right)\right),
$$

with its natural structure of Lie subalgebra of $\mathrm{gl}_{n} \oplus \mathfrak{g l}_{n}$. In fact, the elements $\mathrm{e}_{i j}$ correspond to elements in $\mathfrak{g l}_{n} \oplus \mathfrak{g l}_{n}$ in the following way:

$$
\begin{array}{ll}
\mathrm{e}_{i j} \cong\left(\mathrm{m}_{i j}, 0\right) & \text { for all } i>j, \\
\mathrm{e}_{i j} \cong\left(-\mathrm{m}_{i j},+\mathrm{m}_{i j}\right) & \text { for all } i=j, \\
\mathrm{e}_{i j} \cong\left(0, \mathrm{~m}_{i j}\right) & \text { for all } i<j .
\end{array}
$$

Then the Lie bracket of $\mathrm{gl}_{n}^{*}$ is given by

$$
\begin{array}{ll}
{\left[\mathrm{e}_{i, j}, \mathrm{e}_{h, k}\right]=\delta_{j, h} \mathrm{e}_{i, k}-\delta_{k, i} \mathrm{e}_{h, j}} & \text { for all } i \leq j, h \leq k \text { and } i>j, h>k, \\
{\left[\mathrm{e}_{i, j}, \mathrm{e}_{h, k}\right]=\delta_{k, i} \mathrm{e}_{h, j}-\delta_{j, h} \mathrm{e}_{i, k}} & \text { for all } i=j, h>k \text { and } i>j, h=k, \\
{\left[\mathrm{e}_{i, j}, \mathrm{e}_{h, k}\right]=0} & \text { for all } i<j, h>k \text { and } i>j, h<k .
\end{array}
$$

Note that the elements $(1 \leq i \leq n-1,1 \leq j \leq n)$

$$
\mathrm{e}_{i}=e_{i}^{*}=\mathrm{e}_{i, i+1}, \quad \mathrm{f}_{i}=f_{i}^{*}=\mathrm{e}_{i+1, i}, \quad \mathrm{~g}_{j}=g_{j}^{*}=\mathrm{e}_{j j}
$$

are Lie algebra generators of $\mathrm{gl}_{n}^{*}$. In terms of them, the Lie bracket reads

$$
\left[\mathrm{e}_{i}, \mathrm{f}_{j}\right]=0, \quad\left[\mathrm{~g}_{i}, \mathrm{e}_{j}\right]=\delta_{i j} \mathrm{E}_{i}, \quad\left[\mathrm{~g}_{i}, \mathrm{f}_{j}\right]=\delta_{i j} \mathrm{f}_{j} \quad \text { for all } i, j .
$$

On the other hand, the Lie cobracket structure of $\mathrm{gl}_{n}^{*}$ is given by

$$
\delta\left(\mathrm{e}_{i, j}\right)=\sum_{k=1}^{n} \mathrm{e}_{i, k} \wedge \mathrm{e}_{k, j} \quad \text { for all } i, j=1, \ldots, n,
$$

where $x \wedge y:=x \otimes y-y \otimes x$.

Finally, all these formulæ also provide a presentation of $U\left(\mathfrak{g} \mathfrak{l}_{n}^{*}\right)$ as a co-Poisson Hopf algebra.

A similar description holds for $\mathfrak{I I}_{n}^{*}=\mathfrak{g} \mathfrak{l}_{n}^{*} / Z\left(\mathfrak{g} \mathfrak{l}_{n}^{*}\right)$, where $Z\left(\mathfrak{g} \mathfrak{l}_{n}^{*}\right)$ is the centre of $\mathrm{gl}_{n}^{*}$ generated by $\mathfrak{l}_{n}:=\mathrm{g}_{1}+\cdots+\mathrm{g}_{n}$. The construction is immediate by looking at the embedding $\mathfrak{s l}_{n} \hookrightarrow \mathfrak{g l}_{n}$.

We now turn to the construction of the QDP functor. 
Observation 5.13. $\mathcal{O}_{q}(G)^{\vee}$ (see Definition 4.1 and Section 5.1) is generated, as a unital subalgebra of $\mathcal{O}_{q}(G) \otimes_{\mathbb{k}_{q}} \mathbb{k}(q)$, by the elements

$\Delta_{-}:=(q-1)^{-1}\left(D_{q}^{-1}-1\right), \quad \chi_{i j}:=(q-1)^{-1}\left(x_{i j}-\delta_{i j}\right) \quad$ for all $i, j=1, \ldots, n$, where the $x_{i j}$ 's are the generators of $\mathcal{O}_{q}(G)$. As $x_{i j}=\delta_{i j}+(q-1) \chi_{i j} \in \mathcal{O}_{q}(G)^{\vee}$, we have an obvious embedding of $\mathcal{O}_{q}(G)$ into $\mathcal{O}_{q}(G)^{\vee}$.

Following Definition 4.2, we define

$$
\mathcal{O}_{q}(G / P)^{\vee}:=\left\langle(q-1)^{-1} J_{G / P}^{\mathrm{loc}}\right\rangle=\sum_{n=0}^{\infty}(q-1)^{-n}\left(J_{G / P}^{\mathrm{loc}}\right)^{n} .
$$

We can provide a concrete description of $\mathcal{O}_{q}(G / P)^{\vee}$ :

Proposition 5.14. We have

$$
\mathcal{O}_{q}(G / P)^{\vee}=\mathbb{k}_{q}\left\langle\left\{\mu_{i j}\right\}_{i=r+1, \ldots, n}^{j=1, \ldots, r}\right\rangle / I_{M},
$$

where $\mu_{i j}:=(q-1)^{-1} t_{i j}$ (for all $i$ and $j$ ), $I_{M}$ is the ideal of the Manin relations among the $\mu_{i j}$ 's, and $t_{i j}:=(-q)^{r-j} \Delta_{i j} D_{0}^{-1}$ ( for all $i$ and $j$ ).

Proof. Trivial from definitions and Proposition 5.11.

We want to see explicitly what is $\left.\mathcal{O}_{q}(G / P)^{\vee}\right|_{q=1}$ inside $U\left(\mathfrak{g r}_{n}{ }^{*}\right)$. In other words, we want to understand what is the space that $\mathcal{O}_{q}(G / P)^{\vee}$ is quantizing. We check now by direct inspection that this is $U\left(\mathfrak{p}^{\perp}\right)$, as already prescribed by Theorem 4.8.

\section{Proposition 5.15.}

$$
\left.\mathcal{O}_{q}(G / P)^{\vee}\right|_{q=1}=U\left(\mathfrak{p}^{\perp}\right)
$$

as a subalgebra of $\left.\mathcal{O}_{q}(G)^{\vee}\right|_{q=1}=U\left(\mathrm{gr}_{n}^{*}\right)$, where $\mathfrak{p}^{\perp}$ is the orthogonal subspace to $\mathfrak{p}:=\operatorname{Lie}(P)$ inside $\mathrm{gl}_{n}{ }^{*}$.

Proof. Due to the previous discussion, it is enough to show that

$$
\pi_{G}^{\vee}\left(\mathcal{O}_{q}(G / P)^{\vee}\right)=U\left(\mathfrak{p}^{\perp}\right) \subseteq U\left(\mathfrak{g r}_{n}^{*}\right)=\left.\mathcal{O}_{q}(G)^{\vee}\right|_{q=1} .
$$

To do this, we describe the isomorphism $\left.\mathcal{O}_{q}(G)^{\vee}\right|_{q=1} \cong U\left(\mathrm{gr}_{n}{ }^{*}\right)$ (cf. [14]). According to Remark 5.13, the algebra $\mathcal{O}_{q}(G)^{\vee}$ is generated by the elements

$$
\Delta_{-}:=(q-1)^{-1}\left(D_{q}^{-1}-1\right), \quad \chi_{i j}:=(q-1)^{-1}\left(x_{i j}-\delta_{i j}\right) \quad \text { for all } i, j=1, \ldots, n
$$

inside $\mathcal{O}_{q}(G) \otimes_{\mathbb{k}_{q}} \mathbb{k}(q)$. In terms of these generators, the isomorphism reads

$$
\begin{gathered}
\left.\mathcal{O}_{q}(G)^{\vee}\right|_{q=1} \rightarrow U\left(\mathfrak{g r}_{n}^{*}\right), \\
\overline{\Delta_{-}} \mapsto-\left(\mathrm{e}_{1,1}+\cdots+\mathrm{e}_{n, n}\right), \quad \overline{\chi_{i, j}} \mapsto \mathrm{e}_{i, j} \quad \text { for all } i, j .
\end{gathered}
$$


where we used notation $\bar{X}:=X \bmod (q-1) \mathcal{O}_{q}(G)^{\vee}$. Indeed, from $\overline{\chi_{i, j}} \mapsto \mathrm{e}_{i, j}$ and $(q-1)^{-1}\left(D_{q}-1\right) \in \mathcal{O}_{q}(G)^{\vee}$, one gets $\overline{D_{q}} \mapsto 1$ and $\overline{(q-1)^{-1}\left(D_{q}-1\right)} \mapsto$ $\mathrm{e}_{1,1}+\cdots+\mathrm{e}_{n, n}$. Moreover, the relation $D_{q} D_{q}^{-1}=1$ in $\mathcal{O}_{q}(G)$ implies that $D_{q} \Delta_{-}=$ $-(q-1)^{-1}\left(D_{q}-1\right)$ in $\mathcal{O}_{q}(G)^{\vee}$, whence clearly $\overline{\Delta_{-}} \mapsto-\left(\mathrm{e}_{1,1}+\cdots+\mathrm{e}_{n, n}\right)$, as claimed.

In other words, the specialization $p_{G}^{\vee}: \mathcal{O}_{q}(G)^{\vee} \rightarrow U\left(\mathrm{gr}_{n}^{*}\right)$ is given by

$$
p_{G}^{\vee}\left(\Delta_{-}\right)=-\left(\mathrm{e}_{1,1}+\cdots+\mathrm{e}_{n, n}\right), \quad p_{G}^{\vee}\left(\chi_{i, j}\right)=\mathrm{e}_{i, j} \quad \text { for all } i, j .
$$

If we look at $\widehat{\mathcal{O}_{q}(G)^{v}}$, things are even simpler. Since

$$
D_{q} \in\left(1+(q-1) \mathcal{O}_{q}(G)^{\vee}\right) \subset\left(1+(q-1) \widehat{\mathcal{O}_{q}(G)^{\vee}}\right),
$$

it follows that $D_{q}^{-1} \in\left(1+(q-1) \widehat{\mathcal{O}_{q}(G)^{\vee}}\right)$, and the generator $\Delta_{-}$can be dropped. The specialization map $\widehat{p_{G / P}^{\vee}}$ of course is still described by formulæ as above.

Let us compute $p_{G / P}^{\vee}\left(\mathcal{O}_{q}(G / P)^{\vee}\right)=\widehat{p_{G}^{\vee}}\left(\mathcal{O}_{q}(G / P)^{\vee}\right)$. Recall that $\mathcal{O}_{q}(G / P)^{\vee}$ is generated by the $\mu_{i j}$ 's, with

$$
\mu_{i j}:=(q-1)^{-1} t_{i j}=(q-1)^{-1}(-q)^{r-j} \Delta_{i j} D_{0}^{-1}
$$

for $i=r+1, \ldots, n$ and $j=1, \ldots, r$; thus we must compute $\widehat{p_{G}^{v}}\left(\mu_{i j}\right)$.

By definition, for every $i \neq j$ the element $x_{i j}=(q-1) \chi_{i j}$ is mapped to 0 by $\widehat{p_{G}^{v}}$. Instead, for each $\ell$ the element $x_{\ell \ell}=1+(q-1) \chi_{\ell \ell}$ is mapped to 1 (by $\widehat{p_{G}^{v}}$ again). But then, expanding the $q$-determinants one easily finds that

$$
\begin{aligned}
& \widehat{p_{G}^{v}}\left((q-1)^{-1} \Delta_{i j}\right)=\left((q-1)^{-1} \sum_{\sigma \in \mathcal{S}_{r}}(-q)^{\ell(\sigma)} x_{1 \sigma(1)} \ldots x_{r \sigma(r)}\right) \\
&=\widehat{p_{G}^{v}}\left((q-1)^{-1} \sum_{\sigma \in \delta_{r}}(-q)^{\ell(\sigma)}\left(\delta_{1 \sigma(1)}+(q-1) \chi_{1 \sigma(1)}\right) \ldots\right. \\
&\left.\ldots\left(\delta_{1 \sigma(r)}+(q-1) \chi_{1 \sigma(r)}\right)\right) .
\end{aligned}
$$

The only term in $(q-1)$ in the expansion of $\Delta_{i j}$ comes from the product $\left(1+(q-1) \chi_{11}\right) \ldots\left(1+(q-1) \chi_{r r}\right)(q-1) \chi_{i j} \equiv(q-1) \chi_{i j} \bmod (q-1)^{2} \mathcal{O}(G / P)$.

Therefore, from the previous analysis we get

$$
\begin{aligned}
\widehat{p_{G}^{\vee}}\left((q-1)^{-1} \Delta_{i j}\right) & =\widehat{p_{G}^{\vee}}\left(\chi_{i, j}\right)=\mathrm{e}_{i, j}, \\
\widehat{p_{G}^{\vee}}\left(D_{0}\right) & =\widehat{p_{G}^{\vee}}(1)=1, \\
\widehat{p_{G}^{\vee}}\left(D_{0}^{-1}\right) & =\widehat{p_{G}^{\vee}}(1)=1 .
\end{aligned}
$$


Hence we conclude that $\widehat{p_{G}^{v}}\left(\mu_{i j}\right)=(-1)^{r-j} \mathrm{e}_{i, j}$ for all $1 \leq j \leq r<i \leq n$.

The outcome is that $p_{G / P}^{\vee}\left(\mathcal{O}_{q}(G / P)^{\vee}\right)=U(\mathfrak{h})$, where

$$
\mathfrak{h}:=\operatorname{span}\left(\left\{\mathrm{e}_{i, j} \mid r+1 \leq i \leq n, 1 \leq j \leq r\right\}\right) .
$$

On the other hand, from the very definitions and our description of $\mathfrak{g l}_{n}{ }^{*}$ one easily finds that $\mathfrak{h}=\mathfrak{p}^{\perp}$ for $\mathfrak{p}:=\operatorname{Lie}(P)$. The claim follows.

Proposition 5.16. $\widehat{\mathcal{O}_{q}(G / P)^{\vee}}$ is a left coideal of $\widehat{\mathcal{O}_{q}(G)^{\vee}}$.

Proof. This is the same as Proposition 4.10.

Hence for the quantum Grassmannian we have proved directly the following result:

Theorem 5.17. $\widehat{\mathcal{O}_{q}(G / P)^{v}}$ is a quantum homogeneous $G^{*}$-space, which is an infinitesimal quantization of the homogeneous $G^{*}$-space $\mathfrak{p}^{\perp}$.

5.3. Quantum generalized flag varieties for simple groups as quantum projective homogeneous spaces. We now turn to a more general example of quantum projective space: the quantization of a generalized flag variety for any simple group, following [20]. As before, we begin with a brief description of the classical setting.

Let $G$ be a connected, simply connected, complex simple Lie group and let $\mathrm{g}$ be its Lie algebra. Let $S$ be a subset of simple roots of $g$ and let $\lambda=\sum_{\alpha_{i} \notin S} \omega_{i}$ be a weight of $\mathfrak{g}$, where the $\omega_{i}$ 's are fundamental weights.

Let $V(\lambda)$ be the highest weight representation of $g$ (and of $G$ ) associated with the weight $\lambda$, and let $v_{\lambda}$ be a non-zero highest weight vector of $V(\lambda)$. We have the following morphism of algebraic varieties:

$$
G \rightarrow \mathbb{P}(V(\lambda)), \quad g \mapsto g \cdot v_{\lambda}
$$

This induces a projective embedding of the flag variety $G / P_{S}$ into the projective space $\mathbb{P}(V(\lambda))$, where $P_{S}:=\operatorname{Stab}_{G}\left(v_{\lambda}\right)$ is the parabolic subgroup associated to the set $S$. The graded algebra of regular functions on $G / P_{S}$ relative to this embedding is given by

$$
\mathcal{O}\left(G / P_{S}\right)=\bigoplus_{n \in \mathbb{N}} V(n \lambda)^{*}
$$

where the grading is given by $\mathcal{O}\left(G / P_{S}\right)_{n}:=V(n \lambda)^{*}$ and the multiplication is via the Cartan multiplication (see [12] for more details).

We are now going to identify $\mathcal{O}\left(G / P_{S}\right)$ with a graded subalgebra of $\mathcal{O}(G)$. Indeed, the algebra $\mathcal{O}(G)$ is in (Hopf) duality with $U(\mathrm{~g})$ and it can be thought of as the linear span inside $U(\mathfrak{g})^{*}$ of the functionals $c_{f, v}^{\lambda}: U(\mathfrak{g}) \rightarrow \mathbb{C}$ (the so-called "matrix coefficients") given by

$$
c_{f, v}^{\lambda}(u):=f(u \cdot v) \text { for all } u \in U(\mathfrak{g}), f \in V(\lambda)^{*}, v \in V(\lambda) .
$$


Then $\mathcal{O}\left(G / P_{S}\right)$ can be realized, equivalently, as the subalgebra of $\mathcal{O}(G)$ generated by the $c_{f, v_{\lambda}}^{\lambda}$ 's for all $f \in V(\lambda)^{*}$.

This point of view carries over without changes to the quantum setting: it is considered in [22], [27] and by several others; a quick review of this construction can be found in [20]. The key point is that every finite dimensional representation $V(\mu)$ of $U(\mathfrak{g})$ of highest weight $\mu$ has a (standard) quantization, which is a representation for $U_{q}(\mathrm{~g})$, call it $V_{q}(\mu)$. In particular, one can again define matrix coefficient functionals $c_{f, v}^{\mu}$ - for every $f \in V_{q}(\mu)^{*}$ and $v \in V_{q}(\mu)$ - for all dominant weights $\mu$ of $\mathrm{g}$. Their $\mathbb{k}_{q}$-span inside $U_{q}(\mathrm{~g})^{*}$ is, by definition, the algebra $\mathcal{O}_{q}(G)$, which is a quantization of $\mathcal{O}(G)$. In fact, the latter follows because $V_{q}(\mu)$ respectively $V_{q}(\mu)^{*}$ specializes to $V(\mu)$ respectively $V(\mu)^{*}$ at $q=1$, hence "quantum" and "classical" matrix coefficients bijectively correspond to each other - via $c_{f, v}^{\mu} \mapsto c_{f, v}^{\mu}$, say - under specialization at $q=1$.

For later use, we point out how matrix coefficient behave under the coproduct. For any dominant weight $\mu$ of $\mathfrak{g}$, let $\left\{v_{1}, \ldots, v_{r}\right\}$ be a $\mathbb{k}_{q}$-basis of $V_{q}(\mu)$ with $r=$ $\operatorname{dim}\left(V_{q}(\lambda)\right)$, and let $\left\{f_{1}, \ldots, f_{r}\right\}$ be the dual basis of $V_{q}(\mu)^{*}$. Then every matrix coefficient $c_{f, v}^{\mu}$ has coproduct given by

$$
\Delta\left(c_{f, v}^{\mu}\right)=\sum_{i=1}^{r} c_{f, v_{i}}^{\mu} \otimes c_{f_{i}, v}^{\mu}
$$

(just because $U_{q}(\mathfrak{g})$ is acting on $V_{q}(\mu)$ via matrices!).

From the quantization $\mathcal{O}_{q}(G)$ the group $G$ inherits a Poisson group structure cf. Remark 3.2(2) - for which $P_{S}$ is a Poisson subgroup. On the other hand, let $I_{q}\left(P_{S}\right)$ be the two-sided ideal of $\mathcal{O}_{q}(G)$ generated by the set of matrix coefficients $\left\{c_{f, v}^{\mu} \mid\right.$ for all $n \in \mathbb{N}: \mu \neq n \lambda$ or $\left.v \notin\left(\mathbb{k}_{q} \backslash\{0\}\right) \cdot v_{n \lambda}\right\}$. Then, using (5.5), one easily shows that $I_{q}\left(P_{S}\right)$ is a Hopf ideal of $\mathcal{O}_{q}(G)$; therefore the quotient $\mathbb{k}_{q}$-module and the canonical map

$$
\mathcal{O}_{q}\left(P_{S}\right):=\mathcal{O}_{q}(G) / I_{q}\left(P_{S}\right), \quad \pi: \mathcal{O}_{q}(G) \rightarrow \mathcal{O}_{q}\left(P_{S}\right)
$$

are respectively a quotient Hopf algebra and a Hopf algebra epimorphism. Indeed, this provides a quantization of $P_{S}$ as a Poisson subgroup of $G$, namely $\mathcal{O}_{q}\left(P_{S}\right)$ is a quantum subgroup of $G$ in the sense of Definition 3.3.

In view of the above construction of $\mathcal{O}_{q}(G)$ and of the classical description of $\mathcal{O}\left(G / P_{S}\right)$ in (5.4), we define

$$
\mathcal{O}_{q}\left(G / P_{S}\right)^{\prime}:=\bigoplus_{n \in \mathbb{N}} V_{q}(n \lambda)^{*}=\mathbb{k}_{q} \text {-span of }\left\{c_{f, v_{n \lambda} \lambda}^{n \lambda}\right\}_{f \in V_{q}(n \lambda)^{*}, n \in \mathbb{N}}
$$

where $v_{\lambda}$ is a non-zero element of weight $\lambda$ in $V_{q}(\lambda)$. Then, as the quantum matrix coefficients specialize to the classical ones, comparing (5.7) with (5.4) we see at once that

$$
\mathcal{O}_{q}\left(G / P_{S}\right)^{\prime} /(q-1) \mathcal{O}_{q}\left(G / P_{S}\right)^{\prime} \cong \mathcal{O}\left(G / P_{S}\right)
$$


so that $\mathcal{O}_{q}\left(G / P_{S}\right)^{\prime}$ is a quantization, as a $\mathbb{k}_{q}$-module, of $\mathcal{O}\left(G / P_{S}\right)$.

We are now going to show that this $\mathcal{O}_{q}\left(G / P_{S}\right)^{\prime}$ is in fact a quantum homogeneous space in the sense of Definition 3.18, in particular it can be realized as the space of semi-invariants inside $\mathcal{O}_{q}(G)$ with respect to a suitable quantum section. Indeed, we shall find that $\mathcal{O}_{q}\left(G / P_{S}\right)^{\prime}=\mathcal{O}_{q}\left(G / P_{S}\right)$, where the latter is the space of all semiinvariants (for a suitable quantum section) as in Definition 3.7 and Definition 3.18.

First of all, let $\lambda$ be the dominant weight fixed above, and let $v_{\lambda}$ be the (up to a scalar factor) uniquely determined non-zero element of weight $\lambda$ in $V_{q}(\lambda)$. Fix a $\mathbb{k}_{q}$-basis $\left\{v_{1}, \ldots, v_{r}\right\}$ of $V_{q}(\lambda)$ with $v_{1}=v_{\lambda}$, and let $\left\{f_{1}, \ldots, f_{r}\right\}$ be the dual basis of $V_{q}(\mu)^{*}$ for which we set $f_{\lambda}:=f_{1}$.

Proposition 5.18. The element $c_{f_{\lambda}, v_{\lambda}}^{\lambda}$ is a quantum section in $\mathcal{O}_{q}(G)$.

Proof. Due to Proposition 3.13 (b) we only need to show that $c_{f_{\lambda}, v_{\lambda}}^{\lambda}$ is a pre-quantum section with respect to the setup of (5.6), i.e.,

$$
\Delta_{\pi}\left(c_{f_{\lambda}, v_{\lambda}}^{\lambda}\right)=c_{f_{\lambda}, v_{\lambda}}^{\lambda} \otimes \pi\left(c_{f_{\lambda}, v_{\lambda}}^{\lambda}\right)
$$

But this follows at once from the identity (5.5) applied to the bases chosen above, once we notice in addition that $c_{f_{i}, v_{\lambda}}^{\lambda} \in I_{q}\left(P_{S}\right)$ for all $i \neq 1$.

Proposition 5.19. The space of $c_{f_{\lambda}, v_{\lambda}}^{\lambda}$-semi-invariants of degree 1 inside $\mathcal{O}_{q}(G)$, that is, $\mathcal{O}_{q}\left(G / P_{S}\right)_{1}:=\left\{f \in \mathcal{O}_{q}(G) \mid \Delta_{\pi}(f)=f \otimes \pi\left(c_{f_{\lambda}, v_{\lambda}}^{\lambda}\right)\right\}$, is just the $\mathbb{k}_{q}$-span of $\left\{c_{f, v_{\lambda}}^{\lambda} \mid f \in V_{q}(\lambda)^{*}\right\}$. In other words,

$$
\mathcal{O}_{q}\left(G / P_{S}\right)_{1}=\mathbb{k}_{q} \text {-span of }\left\{c_{f, v_{\lambda}}^{\lambda} \mid f \in V_{q}(\lambda)^{*}\right\}
$$

Proof. This again is immediate as before. Consider any $\mathbb{k}_{q}$-linear combination of several $c_{f, v}^{\mu}$ 's which is semi-invariant of degree 1 with respect to the quantum section $d:=c_{f_{\lambda}, v_{\lambda}}^{\lambda}$. We can assume these $c_{f, v}^{\mu}$ 's to be linearly independent over $\mathbb{k}_{q}$, and so the semi-invariance of their linear combination as a whole also implies the semiinvariance of each of the $c_{f, v}^{\mu}$,'s on its own.

Now assume that a single matrix coefficient $c_{f, v}^{\mu}$ is semi-invariant of degree 1 (with respect to $c_{f_{\lambda}, v_{\lambda}}^{\lambda}$ ). Then (5.5) implies at once that $\mu=\lambda$. Moreover, choosing bases $\left\{v_{1}=v_{\lambda}, v_{2}, \ldots, v_{r}\right\}$ and $\left\{f_{1}=f_{\lambda}, f_{2}, \ldots, f_{r}\right\}$ as before in Proposition 5.18, the identity (5.5) also gives

$$
c_{f, v}^{\lambda} \otimes \pi\left(c_{f_{\lambda}, v_{\lambda}}^{\lambda}\right)=\Delta_{\pi}\left(c_{f, v}^{\lambda}\right)=c_{f, v_{\lambda}}^{\lambda} \otimes \pi\left(c_{f_{\lambda}, v}^{\lambda}\right)+\sum_{i=2}^{r} c_{f, v_{i}}^{\lambda} \otimes \pi\left(c_{f_{i}, v}^{\lambda}\right) .
$$

This forces $c_{f_{i}, v}^{\lambda} \in \operatorname{Ker}(\pi)=I_{q}\left(P_{S}\right)$ for all $i>1$, so that $v \in \mathbb{k}_{q} \cdot v_{\lambda}$, say $v=\kappa v_{\lambda}$ 
for some $\kappa \in \mathbb{k}$, whence eventually

$$
c_{f, v}^{\lambda}=c_{f, \kappa v}^{\lambda}=\kappa c_{f, v}^{\lambda} \in \mathbb{k}_{q} \text {-span of }\left\{c_{f, v_{\lambda}}^{\lambda} \mid f \in V_{q}(\lambda)^{*}\right\} .
$$

This proves that $\mathcal{O}_{q}\left(G / P_{S}\right)_{1}$ is indeed contained in the prescribed $\mathbb{k}_{q}$-span. The converse is clear, just reversing the previous argument.

Proposition 5.20. The space of $c_{f_{\lambda}, v_{\lambda}}^{\lambda}$-semi-invariants of degree $n$ inside $\mathcal{O}_{q}(G)$, that is, $\mathcal{O}_{q}\left(G / P_{S}\right)_{n}:=\left\{f \in \mathcal{O}_{q}(G) \mid \Delta_{\pi}(f)=f \otimes \pi\left(\left(c_{f_{\lambda}, v_{\lambda}}^{\lambda}\right)^{n}\right)\right\}$, is just the $\mathbb{k}_{q}$-span of $\left\{c_{f, v_{n \lambda}}^{n \lambda} \mid f \in V_{q}(n \lambda)^{*}\right\}$. In other words,

$$
\mathcal{O}_{q}\left(G / P_{S}\right)_{n}=\mathbb{k}_{q} \text {-span of }\left\{c_{f, v_{n \lambda}}^{n \lambda} \mid f \in V_{q}(n \lambda)^{*}\right\}
$$

Proof. This follows from an argument which closely mimics the one used in the proof of Proposition 5.19. One takes into account, in addition, the following two remarks:

(a) The vector $v_{\lambda}^{\otimes n}$ has weight $n \lambda$ inside $V_{q}(\lambda)^{\otimes n}$; thus it can be canonically identified with a (non-zero) highest weight vector, say $v_{n \lambda}$, in $V_{q}(n \lambda)$, hence it can be chosen as $v_{1}:=v_{n \lambda}$, the first element of a suitable $\mathbb{k}_{q}$-basis of $V_{q}(n \lambda)$ to be used in that argument;

(b) With notation as above, the $n$-th power function $\left(c_{f_{\lambda}, v_{\lambda}}^{\lambda}\right)^{n}$ inside $\mathcal{O}_{q}(G)$ is nothing but a matrix coefficient again, namely $\left(c_{f_{\lambda}, v_{\lambda}}^{\lambda}\right)^{n}=c_{f_{n \lambda}, v_{n \lambda}}^{n \lambda}$.

These two remarks, drafted into an argument totally similar to the one used for Proposition 5.19, yield the claim.

We are now ready for the main result of this subsection.

Theorem 5.21. Let $\mathcal{O}_{q}\left(G / P_{S}\right)$ be defined as in Definition 3.7, with respect to the quantum section $d:=c_{f_{e}, v_{\lambda}}^{\lambda} \in \mathcal{O}_{q}(G)$. Then $\mathcal{O}_{q}\left(G / P_{S}\right)$ is a quantum projective homogeneous space, namely, it is a quantization of $\mathcal{O}\left(G / P_{S}\right)$, in the sense of Definition 3.18.

Proof. This follows at once by putting together the previous results, i.e., Propositions 5.18, 5.19 and 5.20, and the specialization formula (5.8).

Remark 5.22. (1) With some extra work, one can also show that $\mathcal{O}_{q}\left(G / P_{S}\right)$ is generated, as a graded algebra, in degree 1, i.e., by $\mathcal{O}_{q}\left(G / P_{S}\right)_{1}$.

(2) In this setup of quantum generalized flag varieties one can also apply the QDP, following the general recipe of Section 4.

Indeed, in [20], \$3.4, it is noticed that the quantum section $d:=c_{f_{e}, v_{\lambda}}^{\lambda}$ is $a$ Ore element in $\mathcal{O}_{q}(G)$. Therefore, as pointed out in Section 4.1, we can define the localizations

$$
\mathcal{O}_{q}\left(G / P_{s}\right)\left[\left(c_{f_{e}, v_{\lambda}}^{\lambda}\right)^{-1}\right] \subseteq \mathcal{O}_{q}(G)\left[\left(c_{f_{e}, v_{\lambda}}^{\lambda}\right)^{-1}\right]
$$

and can then apply the QDP, according to Section 4, to this setting. 
5.4. The coisotropic case. One could argue whether the generality we are working with is necessary or not. In this section we will describe how to find families of coisotropic parabolic subgroups inside semisimple algebraic groups with the standard multiplicative Poisson structure.

Such families give rise to smooth Poisson bivectors on the projective quotients which cannot be obtained as quotient by Poisson parabolic subgroups. It would be therefore interesting to investigate whether in such examples it is possible to find and quantize a graded Poisson algebra associated to them as described in Section 2.

We shall start with a low-dimensional example and describe in a very explicit manner the objects we are interested in and then generalize such results.

Let us consider the group $\mathrm{SL}_{2}(\mathbb{C})$ with the standard Poisson algebraic structure, normalized as follows: if $a, b, c, d$ are matrix coefficients in positions $\left(\begin{array}{ll}a & b \\ c & d\end{array}\right)$, we let $\{a, b\}=a b,\{a, c\}=a c,\{b, d\}=b d,\{c, d\}=c d,\{b, c\}=0,\{a, d\}=2 a d$ (this is the normalization opposite to that in [21]). We take the standard parabolic subgroup of upper triangular matrices

$$
P=\left\{\left(\begin{array}{ll}
a & b \\
0 & d
\end{array}\right) \mid a, b, d \in \mathbb{C}\right\}
$$

This is a Poisson subgroup in $\mathrm{SL}_{2}(\mathbb{C})$; thus, the quotient $\mathbb{P}^{1} \mathbb{C} \simeq \mathrm{SL}_{2}(\mathbb{C}) / P$ is endowed with the (homogenous) quotient smooth Poisson bivector $\pi_{0}$.

Let us now consider the following element

$$
g_{\varepsilon}:=\left(\begin{array}{cc}
\sqrt{\varepsilon} & \sqrt{1-\varepsilon} \\
-\sqrt{1-\varepsilon} & \sqrt{\varepsilon}
\end{array}\right), \quad \varepsilon \in[0,1],
$$

and let $P_{\varepsilon}:=g_{\varepsilon} P g_{\varepsilon}^{-1}$. Then $P_{\varepsilon}$ is defined inside the group $\mathrm{SL}_{2}(\mathbb{C})$ by the equation

$$
\sqrt{\varepsilon(1-\varepsilon)}(a-d)=(\varepsilon-1) b+\varepsilon c .
$$

The infinitesimal generators of its Lie algebra are

$$
\begin{aligned}
H_{\varepsilon} & :=g_{\varepsilon} H g_{\varepsilon}^{-1}=(2 \varepsilon-1) H-2 \sqrt{\varepsilon(1-\varepsilon)}\left(X^{+}+X^{-}\right), \\
X_{\varepsilon} & :=g_{\varepsilon} X^{+} g_{\varepsilon}^{-1}=\sqrt{\varepsilon(1-\varepsilon)} H+\varepsilon X^{+}-(1-\varepsilon) X^{-} .
\end{aligned}
$$

It is then easily verified, through the infinitesimal criterion of Proposition 2.2, that $P_{\varepsilon}$ is coisotropic, because

$$
\delta\left(H_{\varepsilon}\right)=H_{\varepsilon} \wedge H, \quad \delta\left(X_{\varepsilon}\right)=X_{\varepsilon} \wedge H .
$$

This means that on $\mathbb{P}^{1} \mathbb{C}$ there is an induced Poisson bivector $\pi_{\varepsilon}$ as quotient $\mathrm{SL}_{2}(\mathbb{C}) / P_{\varepsilon}$. That this Poisson bivector is different from $\pi_{0}$ follows considering the image of the diagonal subgroup of $\mathrm{SL}_{2}(\mathbb{C})$, which induces a single 0-dimensional Poisson leaf with respect to $\pi_{0}$ and an $\mathbb{S}^{1}$-family of 0 -dimensional leaves with respect to $\pi_{\varepsilon}$. 
This phenomenon, as mentioned above, is not only specific for $\mathbb{P}^{1} \mathbb{C}$ but can be, for example, shown to take place for all complex Grassmannians.

Let $G$ be a complex semisimple algebraic group and let $K$ be its real compact form. Up to a factor $l$, which is inessential in what follows, the standard Poisson structure on $G$ can be identified with the one which is automatically defined on it when it is identified with Drinfeld's double of $K$. Let now $H$ be a coisotropic subgroup of $K$ and let us consider the subgroup $H K^{*}$ of $G$ (here $K^{*}=A N$ is the Manin dual of $K$ inside $G$ ). Then $P=H K^{*}$ is parabolic in $G, H=P \cap K$ and $K / H \simeq G / P$ as smooth manifolds. It can be shown quite easily that the coisotropy of $K$ implies the coisotropy of $P$, and furthermore, via Theorem 4.1 in [7], that $K / H$ and $G / P$ are also Poisson diffeomorphic. Thus in order to check whether $P$ is coisotropic it is enough to check whether $P \cap K$ is coisotropic with respect to the standard Poisson structure on the compact real group $K$. There we can rely on results in [7], where a 1-parameter family of coisotropic subgroups $H_{\varepsilon} \subseteq \mathrm{SU}(n)$ was given. Such subgroups induce a 1-parameter family of homogeneous Poisson quotients on complex Grassmannians.

\section{References}

[1] F. Bonechi, N. Ciccoli, R. Giachetti, E. Sorace, and M. Tarlini, The coisotropic subgroup structure of $S L_{q}$ (2, R). J. Geom. Phys. 37 (2001), 190-200. Zbl 0985.16021 MR 1807274

[2] A. Borel, Linear algebraic groups. 2nd ed., Graduate Texts in Math. 126, Springer-Verlag, New York 1991. Zbl 0726.20030 MR 1102012

[3] V. Chari and A. Pressley, A guide to quantum groups. Cambridge University Press, Cambridge 1994. Zbl 0839.17009 MR 1300632

[4] N. Ciccoli, Quantization of co-isotropic subgroups. Lett. Math. Phys. 42 (1997), 123-138. Zbl 0951.17009 MR 1479356

[5] N. Ciccoli and F. Gavarini, A quantum duality principle for coisotropic subgroups and Poisson quotients. Adv. Math. 199 (2006), 104-135. Zbl 1137.58003 MR 2187400

[6] N. Ciccoli and F. Gavarini, A global quantum duality principle for subgroups and homogeneous spaces. Work in progress.

[7] N. Ciccoli and A. J.-L. Sheu, Covariant Poisson structures on complex Grassmannians. Comm. Anal. Geom. 14 (2006), 443-474. Zbl 1108.53043 MR 2260719

[8] C. de Concini, D. Eisenbud, and C. Procesi, Young diagrams and determinantal varieties. Invent. Math. 56 (1980), 129-165. Zbl 0435.14015 MR 558865

[9] R. Fioresi, Quantum deformation of the Grassmannian manifold. J. Algebra 214 (1999), 418-447. Zbl 0955.17007 MR 1680557

[10] R. Fioresi, A deformation of the big cell inside the Grassmannian manifold $G(r, n)$. Rev. Math. Phys. 11 (1999), 25-40. Zbl 0955.16034 MR 1668067

[11] R. Fioresi, Quantum deformation of the flag variety. Comm. Algebra 27 (1999), 5669-5685. Zbl 0955.17008 MR 1713061 
[12] R. Fioresi and C. Hacon, Quantum coinvariant theory for the quantum special linear group and quantum Schubert varieties. J. Algebra 242 (2001), 433-446. Zbl 0990.20032 MR 1848953

[13] F. Gavarini, The quantum duality principle. Ann. Inst. Fourier (Grenoble) 52 (2002), 809-834. Zbl 1054.17011 MR 1907388

[14] F. Gavarini, The global quantum duality principle. J. Reine Angew. Math. 612 (2007), 17-33. Zbl 05267807 MR 2364072

[15] F. Gavarini, The global quantum duality principle: theory, examples, and applications. Preprint 2003. arXiv:math/0303019

[16] K. R. Goodearl and T. H. Lenagan, Quantized coinvariants at transcendental q. In Hopf algebras in noncommutative geometry and physics, Lecture Notes in Pure and Appl. Math. 239, Dekker, New York 2005, 155-165. Zbl 1083.16024 MR 2106928

[17] P. Griffiths and J. Harris, Principles of algebraic geometry. Wiley Classics Library, John Wiley \& Sons Inc., New York 1994. Zbl 0836.14001 MR 1288523

[18] R. Hartshorne, Algebraic geometry. Graduate Texts in Math 52, Springer-Verlag, New York 1977. Zbl 0367.14001 MR 0463157

[19] A. Klimyk and K. Schmüdgen, Quantum groups and their representations. Texts Monographs Phys., Springer-Verlag, Berlin 1997. Zbl 0891.17010 MR 1492989

[20] S. Kolb, The AS-Cohen-Macaulay property for quantum flag manifolds of minuscule weight. J. Algebra 319 (2008), 3518-3534. Zbl 05300819 MR 2408329

[21] L. I. Korogodski and Y. S. Soibelman, Algebras of functions on quantum groups: Part I. Mathematical Surveys and Monographs 56, Amer. Math. Soc., Providence, RI, 1998. Zbl 0923.17017 MR 1614943

[22] V. Lakshmibai and N. Reshetikhin, Quantum flag and Schubert schemes. In Deformation theory and quantum groups with applications to mathematical physics (Amherst, MA, 1990), Contemp. Math. 134, Amer. Math. Soc., Providence, RI, 1992, 145-181. Zbl 0792.17012 MR 1187287

[23] V. Lakshmibai, C. Musili, and C. S. Seshadri, Geometry of G/ P. IV: Standard monomial theory for classical types. Proc. Indian Acad. Sci. Sect. A Math. Sci. 88 (1979), 279-362. Zbl 0447.14013 MR 553746

[24] J. H. Lu, Multiplicative and affine Poisson structures on Lie groups. Ph.D. thesis, University of California, Berkeley, 1990. http://hkumath.hku.hk/ jhlu/publications.html

[25] Yu. I. Manin, Topics in noncommutative geometry. Princeton University Press, Princeton, NJ, 1991. Zbl 0724.17007 MR 1095783

[26] S. Montgomery, Hopf algebras and their actions on rings. CBMS Regional Conf. Ser. in Math. 82, Amer. Math. Soc., Providence, RI, 1993. Zbl 0793.16029 MR 1243637

[27] Ya. S. Soibelman, On the quantum flag manifold. Funktsional. Anal. i Prilozhen. 26 (1992), no. 3. 90-92; English transl. Funct. Anal. Appl. 26 (1992), 225-227. Zbl 0820.17017 MR 1189033

[28] E. Taft and J. Towber, Quantum deformation of flag schemes and Grassmann schemes. I. A $q$-deformation of the shape-algebra for GL(n). J. Algebra 142 (1991), 1-36. Zbl 0739.17007 MR 1125202 
[29] S. Zakrzewski, Poisson homogeneous spaces. In Quantum groups: formalism and applications (Karpacz, 1994), Polish Scientific Publishers PWN, Warsaw 1995, 629-639. Zbl 0880.53043 MR 1648009

Received February 5, 2008

N. Ciccoli, Dipartimento di Matematica, Università di Perugia, Via Vanvitelli 1, 06123 Perugia, Italy

E-mail: ciccoli@dipmat.unipg.it

R. Fioresi, Dipartimento di Matematica, Università di Bologna, Piazza di Porta San Donato 5, 40127 Bologna, Italy

E-mail: fioresi@dm.unibo.it

F. Gavarini, Dipartimento di Matematica, Università di Roma “Tor Vergata”, Via della ricerca scientifica 1, 00133 Roma, Italy

E-mail: gavarini@mat.uniroma2.it 